\title{
Interactive Graphics Applications with OpenGL Shading Language and Qt
}

\author{
João Paulo Gois \& Harlen C. Batagelo \\ Centro de Matemática Computação e Cognição \\ Universidade Federal do ABC \\ Santo André, Brazil \\ http://professor.ufabc.edu.br/ \{joao.gois,harlen.batagelo $\}$
}

\begin{abstract}
Qt framework allows the easy development of professional cross-platform graphics applications using $\mathrm{C}++$. Qt provides the QtOpenGL Module that makes easy the development of hardware-accelerated graphics applications using OpenGL and OpenGL Shading Language (GLSL). With Qt, matrices, vectors, vertex buffer objects, textures, shader programs and UI components are integrated by classes in the object-oriented paradigm and intercommunicate by the $Q t$ mechanism of signals/slots. The goal of this survey is to detail the development of interactive graphics applications with OpenGL and Qt. Along with it, we compare features of QtOpenGL Module with those of GLU/GLUT libraries, as the latter is traditionally used in text books and computer graphics courses.
\end{abstract}

\section{INTRODUCTION}

OpenGL [1] is the most popular cross-platform industry standard API for writing $2 \mathrm{D}$ and $3 \mathrm{D}$ interactive graphics applications such as for CAD, video games, scientific visualization, information visualization and virtual reality. Traditionally, course syllabus and textbooks of computer graphics are approached with OpenGL and its cross-platform window management library GLUT [2].

GLUT provides resources to control windows associated to OpenGL contexts and to perform I/O communication (mouse and keyboard devices) with the operating system. Although GLUT is broadly popular for educational purposes, it is quite limited to produce full-featured applications because its set of features is relatively restricted.

In this sense, the Qt framework [3], [4], [5] is an alternative for developing professional interactive graphics applications based on OpenGL and its shading language GLSL. Qt is opensource, cross-platform and available under different licenses, including GNU LGPL 2.1, GNU GPL 3.0 and a commercial developer license. Prominent corporations have employed Qt in their projects, e.g., Autodesk, Adobe, Skype (now part of Microsoft), Wolfram, DreamWorks, Google, Lucasfilm, Samsung, Siemens, Volvo and Walt Disney Animation Studios [6].

Qt offers the QtOpenGL Module [7]: a full-feature set of containers to make easy the development of graphics application using OpenGL/GLSL. Qt integrates to GLSL through QShader and QShaderProgram classes that require small coding effort to compile, link and bind shader programs to applica-

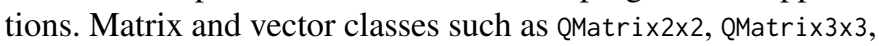

QMatrix4x4, QVector2D, QVector3D, QVector4D and QQuaternion allow for operating with matrices, vectors and quaternions, respectively. In particular, Qmatrix $4 \times 4$ provides methods for orthogonal and perspective projections and for camera settings. The contents of these classes can be easily bound to shaders as storage qualifiers (attribute or uniform) using different shader types, e.g. vec2, vec3, vec4, mat2, mat3 and mat4.

For texture manipulation, image files can be loaded to the application using the Qt class QImage. Through the Qt class QGLWidget, such images can be directly bound to the OpenGL texture units used in the shader programs.

The use of some recent OpenGL extensions such as Vertex Buffer Objects, Frame Buffer Objects and Pixel Buffer Objects, may make the source code easily cluttered and non-intuitive, specially for beginner developers. In its turn, Qt provides classes that encapsulate most details of the many extensions available for shader programming, thus producing code that is cleaner and easier to maintain.

\section{A few requirements}

There are only a few basic requirements to take the best advantages of this survey. The reader should have some familiarity with object-oriented programming, $\mathrm{C}++$ preferred. Also, previous knowledge on computer graphics is required. In particular, the reader should be familiar with foundations of geometric transformations, viewing, lighting and shading [8], [9]. Since we exemplify applications with GLSL, familiarity with this shading language is desirable [10], [11].

\section{Structure of the presentation}

In the next section we guide the reader through the development of an interactive OpenGL/GLSL application with Qt. First we start up a Qt project with minimal support to OpenGL. Next we show how to visualize surface meshes loaded from a file and how to rotate and scale them with a virtual trackball. Different shader effects (Gouraud shading, Phong shading, texture mapping and normal mapping) and simple Qt UI components are also introduced. After presenting this interactive application, we then discuss how to improve its interface with richer UI components (Sec. IIIT). Finally, we conclude our presentation (Sec. IV) and describe directions for further graphics-based Qt applications (Sec. V). 


\section{Creating an OpenGL/GLSL Application with QT}

\section{A. Starting up a Qt project}

The Qt libraries and tools are enclosed in the Qt SDK, available in the Qt website [3] for Windows, Linux and Mac OS X. In this work we develop our application under Qt Framework version 4.8 using the Qt Creator IDE version 2.4.1 (Figure 11) for Linux/Ubuntu. In the following we will detail this process. First we will create a Qt project to our application. After that we will design the UI, write the C++ and GLSL codes and manage the assets of our application.

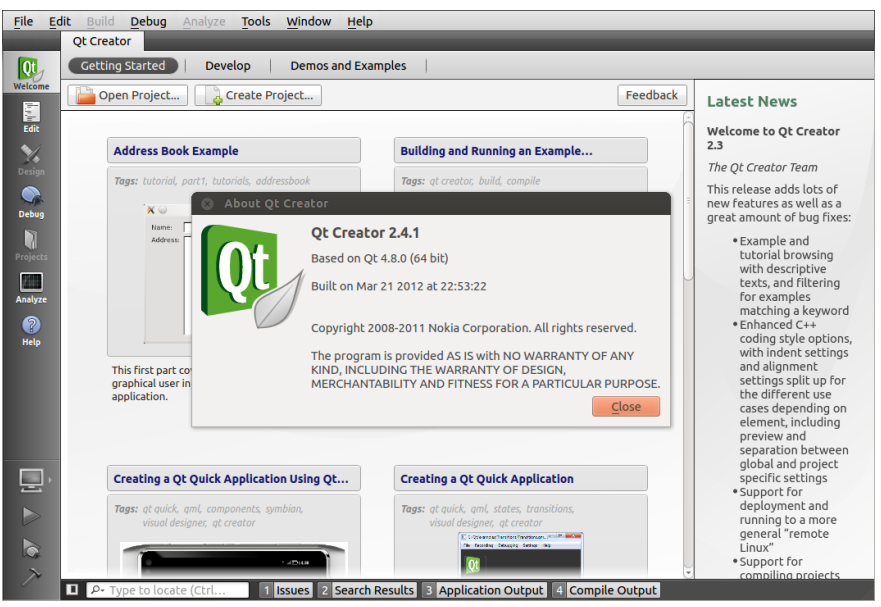

Fig. 1. Interface of the Qt Creator IDE.

Tip 1. To maintain the coherence with the Qt style, we follow the Qt Coding Guidelines [12] in our C++ files.

Let us start by creating a Qt project. Clicking at File $\rightarrow$ New File or Project on the Qt Creator menu bar, we see that Qt offers some project templates. In our case, we choose $Q t G u i$ Application (Fig. 2-(a)), which creates a minimal application for desktop with a main window, a menu bar, a tool bar, a status bar and a central widget (Fig. 3-(b)).

Following steps depicted in Fig. 2, first we select the $Q t$ Gui Application option (Fig. 2-(a)), second we define the project name, as well as its path (Fig. 2.(b)), third we select Desktop as the build target and Use Shadow Building to create a separate directory for storing the compiled sources, objects and executable files (Fig. 2. (c)). Finally, we specify details of the main window interface class as shown in Fig. 2.(d). Qt automatically generated four files:

- myqtglproject.pro: this file, depicted in Listing 1, contains the configurations of the Qt project. It lists the source files of the project, as well as the modules used. Initially, only two Qt modules are added: core and gui. In order to enable QtOpenGL Module, we add opengl in myqtglproject.pro, as depicted in Listing 2.

- mainwindow.\{h,cpp\}: these are the files for the MainWindow class (Listings 3 and 47). In the declaration of this class, we notice the Qt macro Q_OBJECT, which is automatically declared under the private access modifier.

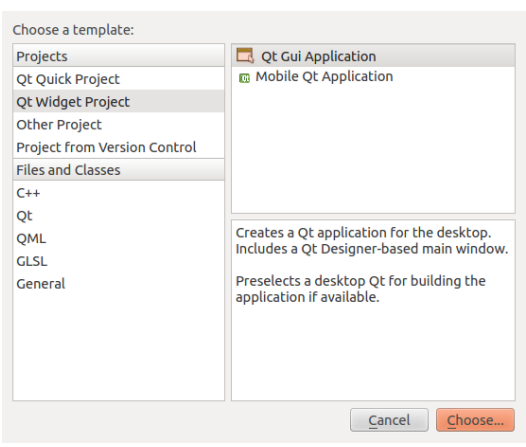

(a)

Introduction and Project Location

This wizard generates a Qt4 GUI application project. The application derives by default from QApplication and includes an empty widge.

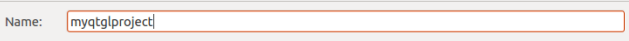

Create in: /home/pgoois/Dropbox/colaboradores/harlen/tutorial_sibgrapizo12/qtprojects Browse.... Use as default project location

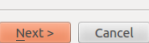

(b)

Target Setup

Qt creator can set up the following targets for project myatglproject.

\begin{tabular}{l|l}
\hline 밀 Desktop Details &
\end{tabular}

Create Build Configurations: For Each Qt Version One Debug And One Release : $\checkmark$ use shadow Building

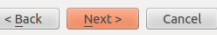

(c)

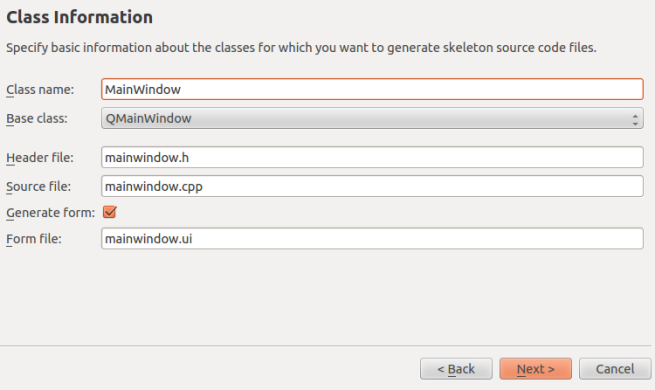

(d)

Fig. 2. Step-by-step to start up a Qt GUI Project. 


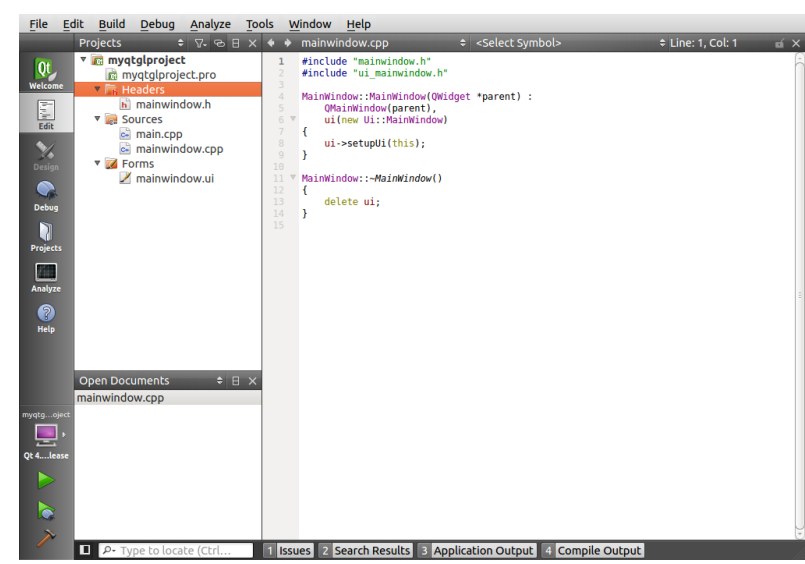

(a)

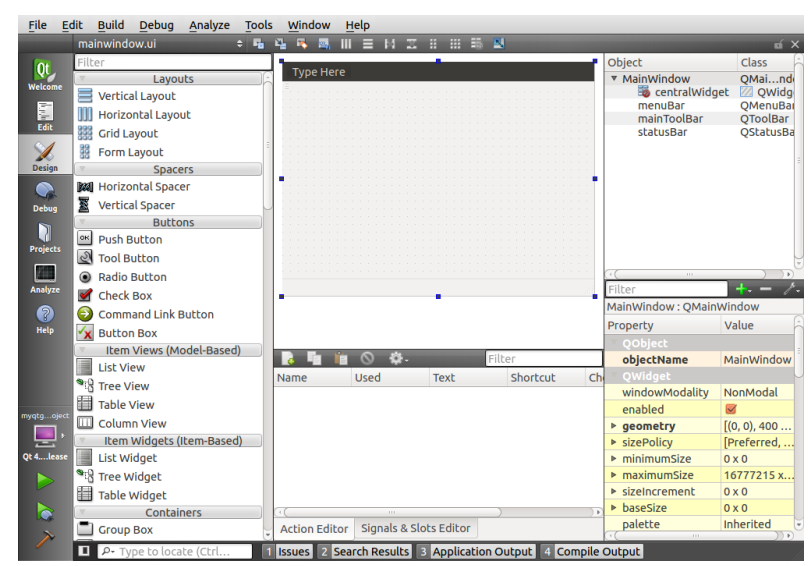

(b)

Fig. 3. Qt Creator: (a) the Edit mode, (b) the Design mode.

This macro is mandatory for any class that implements the Qt concept of Signals and Slots (presented in Sec. III-D). For our application it will not be required to make changes on these files.

- main.cpp: this file contains the $\mathrm{C}++$ main function (Listing 5. We will keep main.cpp as simple as we can. We will only insert further code on it to format details of OpenGL context, for instance, to use antialiasing. It will be detailed in Section $\Pi-N$

- mainwindow.ui: this file contains the XML description of the user interface. This XML file is translated by the Qt User Interface Compiler (uic) to a $\mathrm{C}++$ header file that contains declarations of object widgets used by the application. Fortunately, we do not need to write directly the XML file. Instead, we can edit the user interface using the Qt WYSIWYG editor: the Design mode. When double-clicking on the mainwindow.ui file listed in the Projects Pane, Qt Creator switches to the Design mode (Fig. 3-bottom). The interface of Design mode contains (Fig. 4p: (A) components to design the UI; (B) UI WYSIWYG editor; (C) hierarchy of currently created UI components; (D) properties of the UI components. To come back to the text editor, the Edit mode, click on the
Edit button in the leftmost pane of Qt Creator.

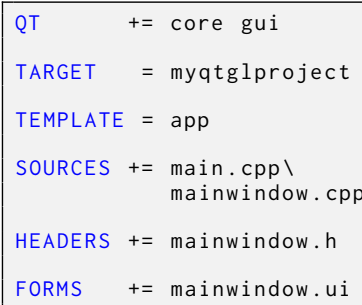

Listing 1. File myqtglproject.pro: initial configuration of the Qt project.

\begin{tabular}{l}
\hline QT $\quad+=$ core gui opengl \\
$\begin{array}{l}\text { Listing } 2 \text {. File myqtglproject.pro: including QtOpenGL Module at } \\
\text { myqtglproject.pro. }\end{array}$
\end{tabular}

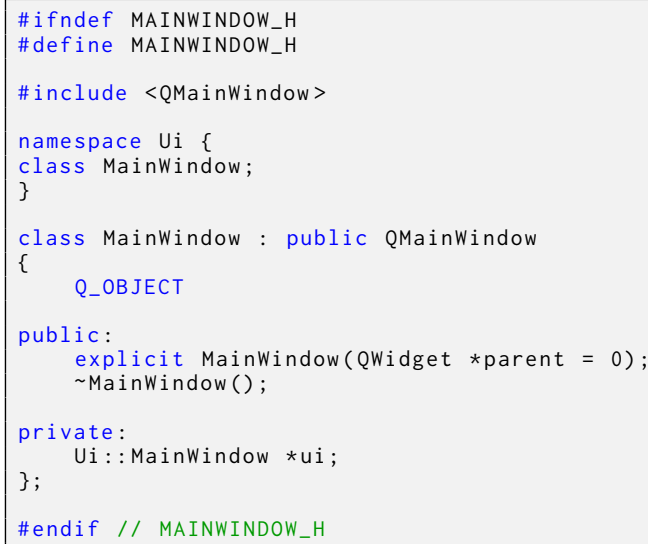

Listing 3. File mainwindow.h: automatically generated by Qt Creator.

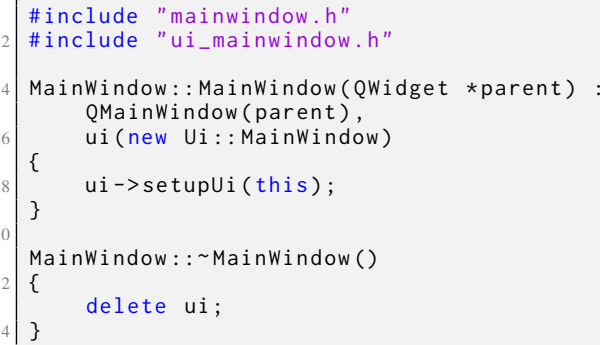

Listing 4. File mainwindow.cpp: automatically generated by Qt Creator.

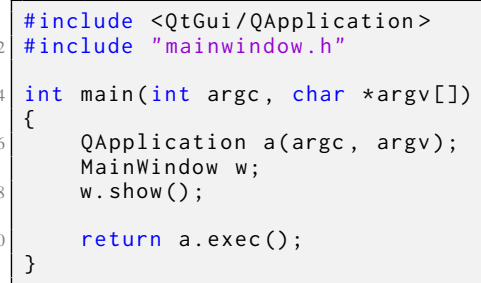

Listing 5. File main.cpp: automatically generated by Qt Creator.

Tip 2. It is also possible to include non-Qt libraries in the $Q t$ project. We show an example in Listing 6 where we include hypothetical libraries foo and bar in the pro file. In this case, we assume they are located at /usr/local/lib/foo and their header files are at/usr/loca/include/foo. 
INCLUDEPATH $+=/$ usr/local/include/foo/

LIBS $+=-$ L/usr/local/lib/foo/ -lfoo -lbar

Listing 6. File myqtglproject.pro: including non-Qt libraries in the Qt project.

Before building the project, the qmake [13] tool uses the configuration from the .pro file to automatically generate, in the shadow building directory, the Makefile file for the target platform. qmake is also able to generate project files for Apple Xcode and Microsoft Visual Studio. Further details about qmake and its configuration variables are found on [13].

Five UI objects are automatically generated by Qt Creator (Fig. 4-(C)): MainWindow, of class QMainWindow, is the main window object and the root node of the hierarchy of UI objects. Its children are centralWidget of class QWidget, menuBar of class QMenuBar, mainToolBar of class QToolBar and statusBar of class QStatusBar. We can use the Design mode to create new child objects. Fig. 5 lists all UI objects of our application. They will be detailed along the next sections.

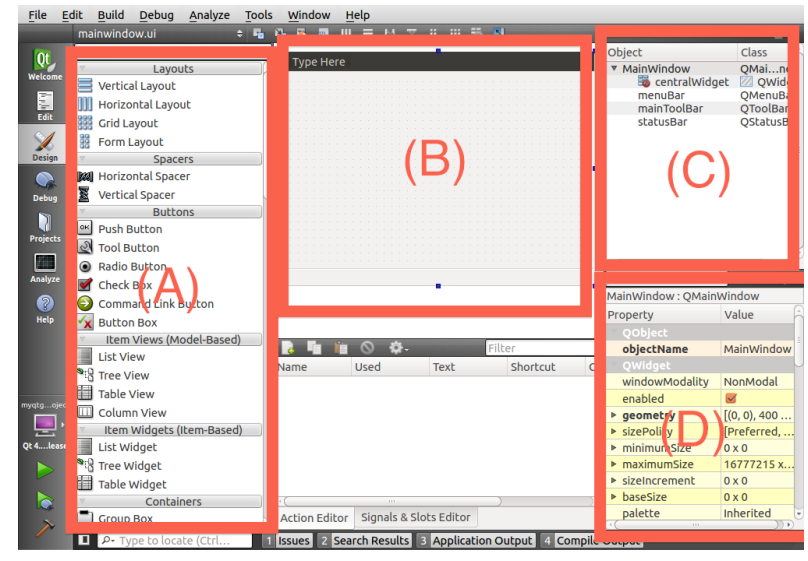

Fig. 4. Qt Creator (Design mode): (A) components to design the UI, (B) UI WYSIWYG editor, (C) hierarchy of currently created UI components and (D) properties of UI components.

\begin{tabular}{|c|l|}
\hline Object & Class \\
\hline MainWindow & QMainWindow \\
\hline $\begin{array}{c}\text { centralWidget } \\
\text { checkBox } \\
\text { pushButton }\end{array}$ & QWidget \\
Q widget & QPh....Box \\
$\nabla$ menuBar & QLWidget \\
$\nabla$ menuFile & QMenuBar \\
actionOpen & QAction \\
mainToolBar & QToolBar \\
statusBar & QStatusBar
\end{tabular}

Fig. 5. UI objects of our application: some of them were automatically generated by Qt Creator and others were added using the Design mode.

So far we learnt how to generate a Qt Project. Our next step is to create an OpenGL interactive application in this project. We show how to do it in a two-step process: in the first, we use the Edit mode to create a custom class that supports OpenGL functionalities (Sec. II-B). In the second, we use the Design mode to associate a widget to an object of the previously created custom class (Sec. II-C) for displaying the OpenGL scene.

B. Edit mode: extending the class QGLWidget to our OpenGL application

The Qt class QWidget provides the widget base for all UI objects. In particular, it is the base for the widget class QGLWidget used for rendering OpenGL graphics [14]. QGLWidget provides virtual methods that should be implemented to perform common OpenGL tasks. Three of them are the most used and will be implemented in our applications:

- paintGL(): renders the OpenGL scene whenever the widget needs to be repainted. It is equivalent to the callback function registered through the GLUT function glutDisplayFunc().

- resizeGL (int width, int height): handles the resizing of the OpenGL window and repaints it. It is equivalent to the callback function registered through the GLUT function glutReshapeFunc().

- initializegL(): it is called whenever the widget is assigned to a new OpenGL context. It is intended to contain the OpenGL initialization code that comes before the first call to paintGL() or resizeGL().

We will define these methods in our custom class GLWidget, derived from the base class QGLWidget. An easy approach to create a new $\mathrm{C}++$ class in a Qt project is as follows: click at File $\rightarrow$ New File or Project to open a dialog window (Fig. 6-(a)). Choose the $\mathrm{C}++$ class template and fill-in all fields (Fig. 6-(b)). Our base class is a QGLWidget and inherits from QWidget. In Fig. 6-(c) we finish the class creation. Qt Creator automatically creates both .h and .cpp files for the GLWidget class (Listings 7 and 8). Notice that Qt does not automatically declare virtual methods of the parent class. We add the code for paintGL(), resizeGL() and initializeGL() on the class files, as depicted in Listings 9 and 10 .

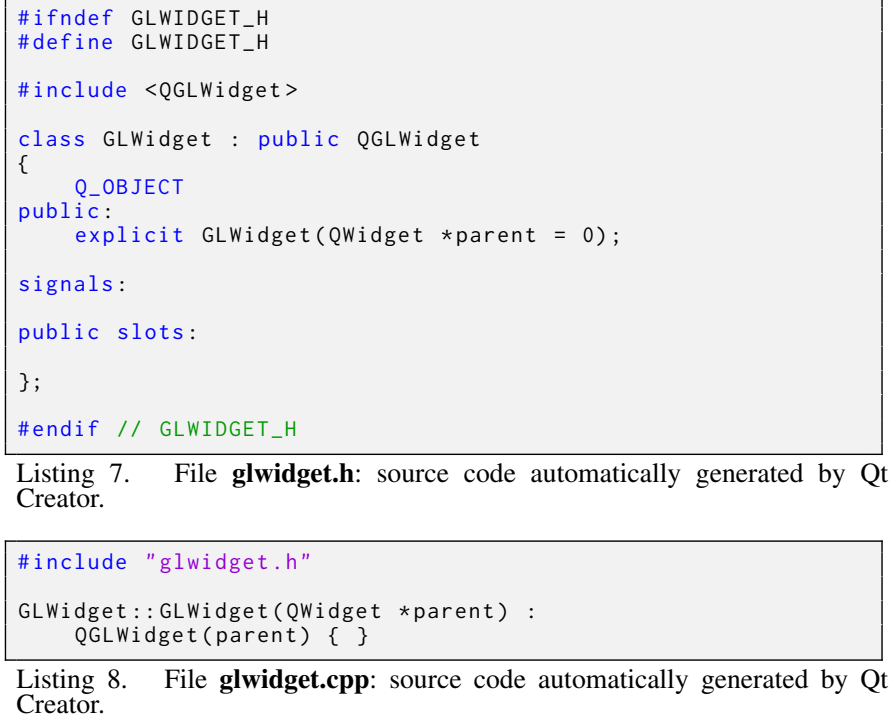

Listing 8. File glwidget.cpp: source code automatically generated by Qt Creator. 


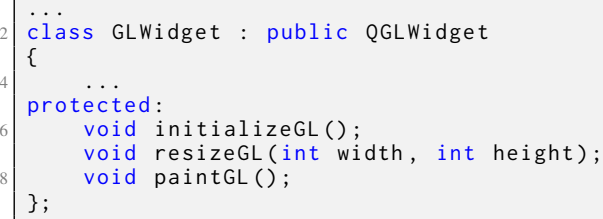

Listing 9. File glwidget.h: declaration of three methods in class GLWidget.

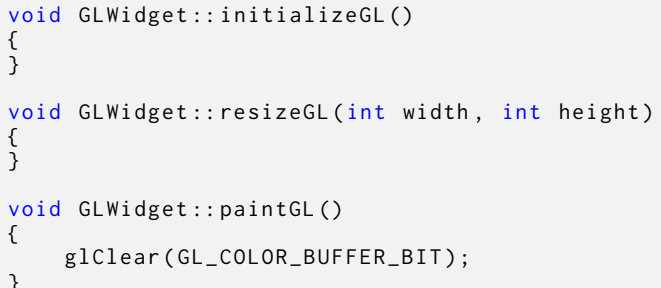

Listing 10. File glwidget.cpp: first implementations.

Tip 3. There is an include file for each class of the QtOpenGL Module. Several of these classes are used in our project. Instead of including each one, we can simply write \#include $<$ QtOpenGL $>$ at glwidget. h. The complete listing of glwidget. $\mathrm{h}$ is in Appendix $A$

\section{$C$. Design mode: promoting $a$ QWidget to GLWidget}

Now we need to add to the main window a widget that displays the OpenGL graphics handled by our custom class GLWidget. However, GLWidget is not in the list of UI components available in the Design mode (Fig. 4. (A)). To overcome that we will initially use the base widget QWidget of GLWidget as a placeholder and then promote it to GLWidget.

Following the steps in Fig. 7, first we drag and drop an Widget onto the object centralWidget (Fig. 77-(a)), creating an object named widget of class Qwidget. As we need that the placeholder widget have the specialization of GLWidget, we apply a Promote Operation: right-click on the widget object and click on Promote to ... (Fig. 7f-(b)). At the new dialog window, type GLWidget at Promoted class name and select QWidget at Base class name. Click on Add and finally click on Promote (Fig. 가(c)).

Tip 4. By default, widget has a fixed size. In order to automatically adjust its size when MainWindow is resized, we must set the sizePolicy - Horizontal/Vertical property of the widget (Fig. 44(D)) from the default option - Preferred - to Expanding. Other resize policies are also available.

Tip 5. UI components can be consistently arranged within widgets using Qt layout styles [15]. We can choose, for instance, the Vertical, Horizontal or Grid layouts. In our application, centralWidget is laid out vertically. In Design mode, this is done by clicking at centralwidget and then either pressing Ctrl + L or clicking on the icon III in the Qt Creator toolbar.

To run our application, either click on the icon Run $\square$ in the leftmost Qt Creator pane or press Ctrl+R.

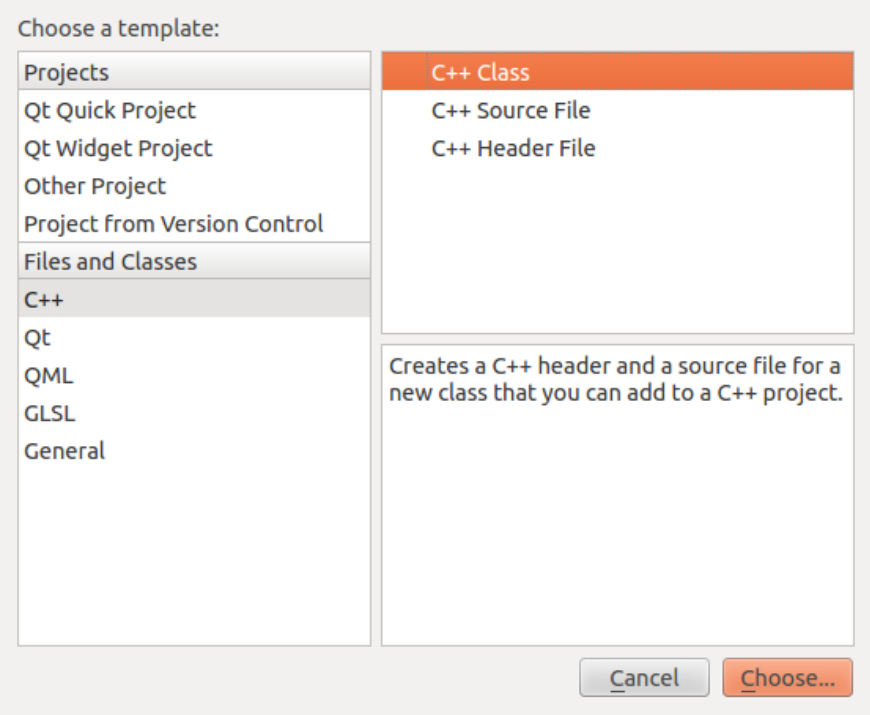

(a)

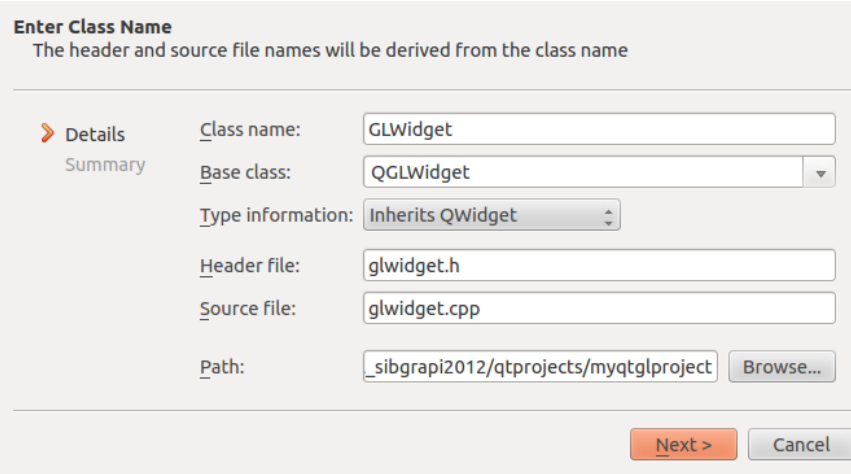

(b)

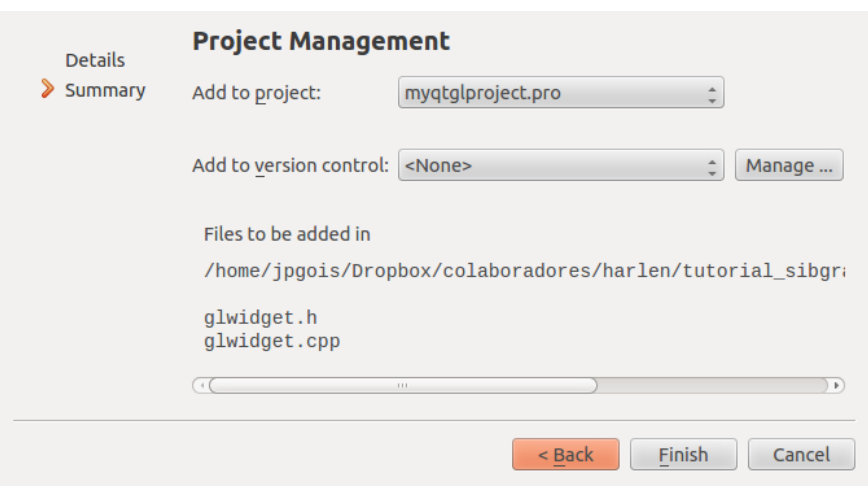

(c)

Fig. 6. Creating the class GLWidget extended from QGLWidget: on (a) we select C++ Class, on (b) we name the class, define the base class QGLWidget and set type information Inherits QWidget. On (c) we finalize.

\section{Introducing Signals and Slots}

OpenGL applications using GLUT [2] make use of the concept of callback functions to handle window events, input device events and timers. With Qt, callback functions can be avoided as it provides not only methods to handle events (Sec. III-I but also a mechanism for object intercommunication, 


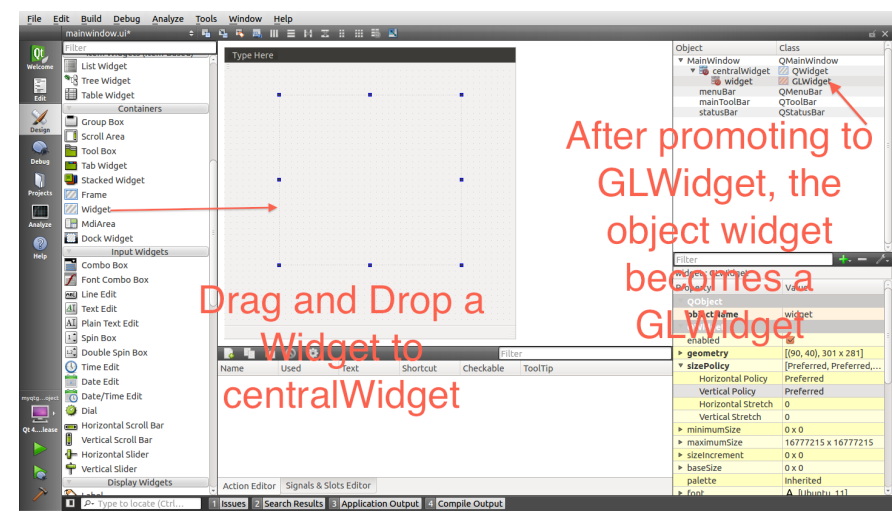

(a)

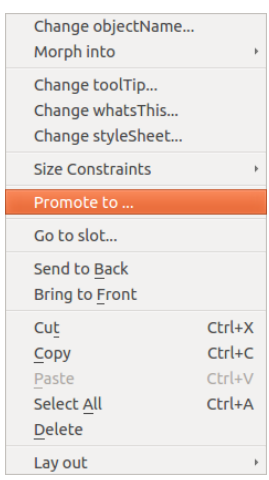

(b)

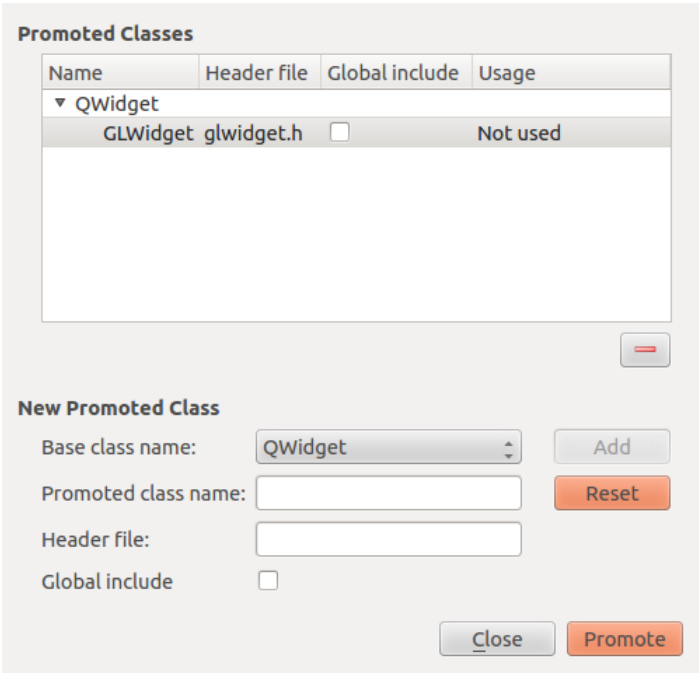

(c)

Fig. 7. Promoting the class QWidget to GLWidget.

named Signals and Slots [16].

A signal is emitted when an event associated to some sender object is triggered, for instance, when a push button is clicked in the UI. A slot, in turn, is a method of a receiver object which is called in response to a particular signal. Slots can be qualified as private, protected or public. Signals are implicitly declared as protected. Differently from callback functions, signal and slots are type-safe, i.e., the signature of a specific sender signal must match the signature of the receiver slot. The complete description of signals and slots is found on [16].

First we will show how to connect a pre-defined signal to a pre-defined slot for creating an Exit push button that quits the application. At Design mode, drag and drop a QPushButton to centralWidget and change the text property of this newly created button from PushButton to Exit (Fig. 85-(a)).

The signal corresponds to the event of pressing the button. It is associated to the method clicked() of the object pushButton of QPushButton class. The slot quits the application. It is associated to the method close() of the object MainWindow of QMainWindow class. Qt Creator provides different ways to make this signal/slot connection. We will show how to do that in the Design mode.

Click at the Signal \& Slots Editor tab (highlighted at Fig. 8.(b)). At this moment, there is no signal/slot connection as shown in Fig. 8 (a). To add our first signal/slot connection, click on the $\$$ icon. A row with four combo boxes appears, which are changed as follows:

- Sender combo box: select pushButton;

- Signal combo box: select the signal clicked() of pushButton;

- Receiver combo box: select MainWindow;

- Slot combo box: select the signal close() of MainWindow.

At this moment we had concluded our first signal/slot connection.

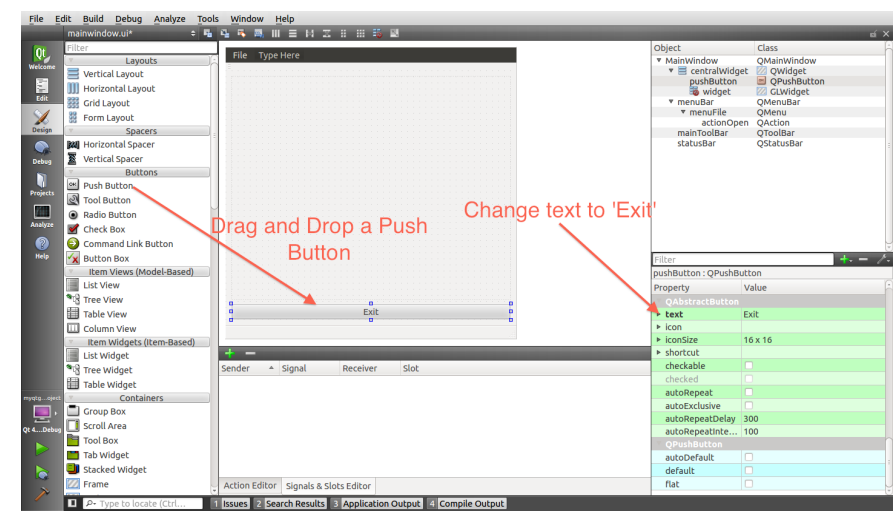

(a)

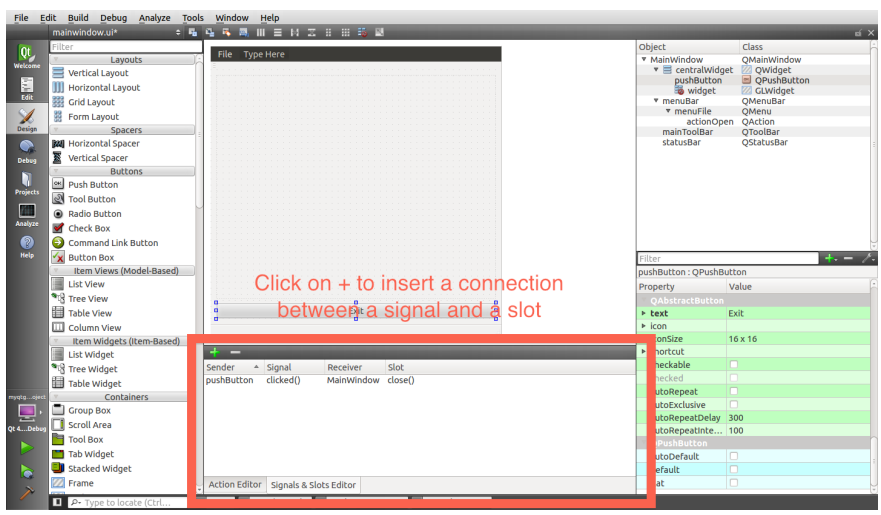

(b)

Fig. 8. Signal/slot connection: on (a) we create a button, whereas on (b) we make a signal/slot connection. 
Now we will exemplify another signal/slot connection, where the signal is pre-defined but the slot is customized. We will create a checkbox button (Fig. 9) that toggles the background color of the widget between white and black using OpenGL commands. The signal is the action of toggling the checkbox, whereas the slot is a method of GLWidget that changes the OpenGL clear color. Before we make the signal/slot connection, let us create the custom slot:

1) In Edit mode, declare the custom slot as shown in Listing 11 ,

2) In Edit mode, define the custom slot as shown in Listing 12 ,

3) In Design mode, insert the new signature of the custom slot to the Signals/Slots dialog of the receiver object: to open this dialog, right-click on widget and select Change signals/slots... (Fig. 10.(a)). Note that this option is only available to our custom OpenGL widget. Now click at the plus button located right below the Slots list widget (Fig. 10.(b)) and enter the signature of the custom slot: toggleBackgroundColor(bool). Click OK to finish.

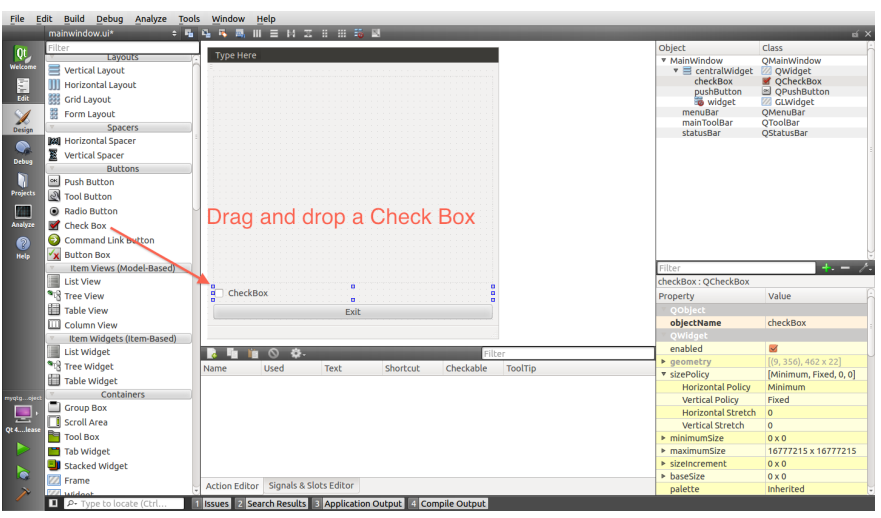

(a)

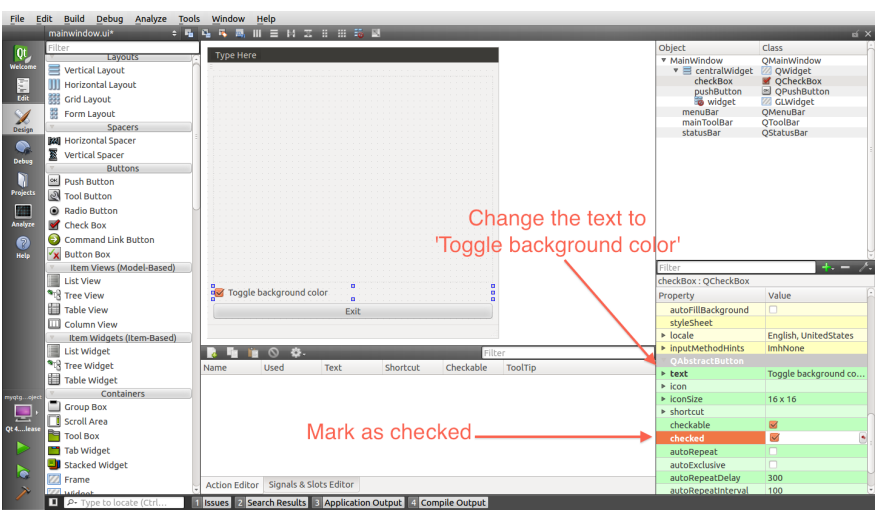

(b)

Fig. 9. Creating a checkbox button: on (a) we drag and drop a Check Box to the centralwidget. On (b) we rename it and set its initial state to checked.

After these steps we proceed as in the previous signal/slot connection example:

- Sender combo box: select checkBox;

- Signal combo box: select the signal toggled(bool) of checkBox;

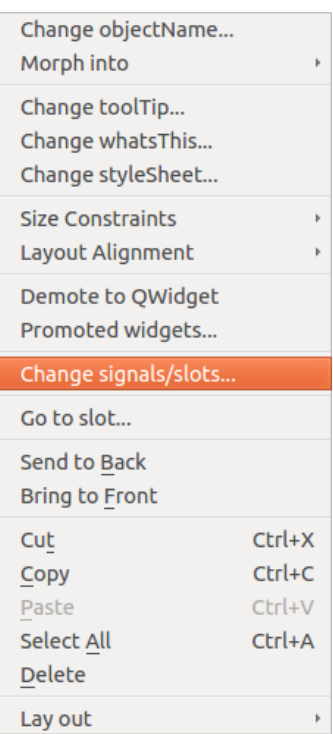

(a)

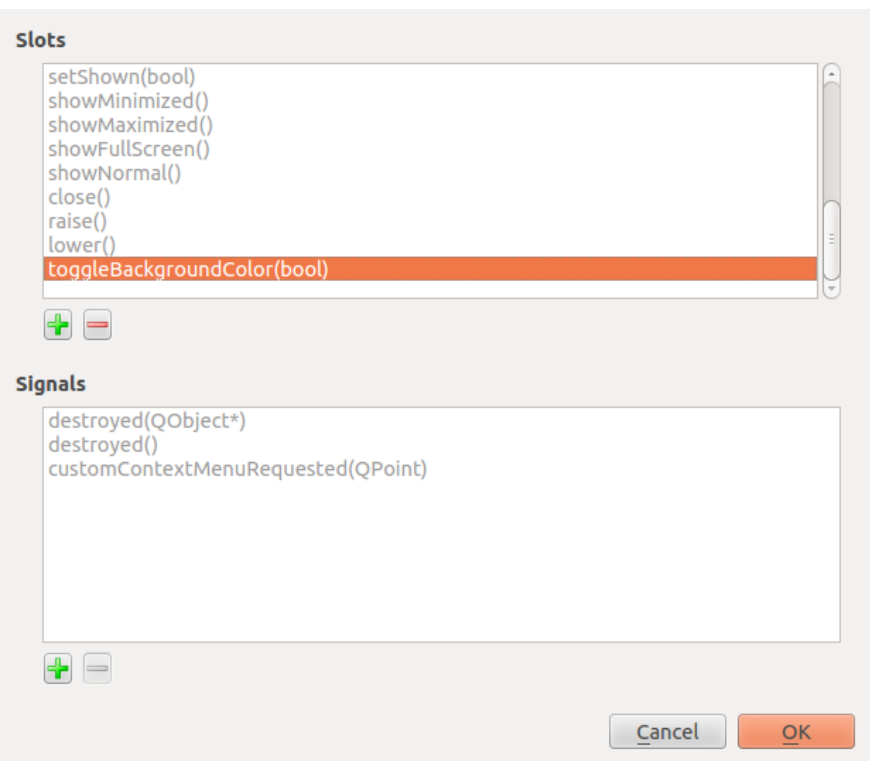

(b)

Fig. 10. Inserting the new signature of the custom slot to the Signals/Slots dialog: the menu on (a) is displayed after a right-click on the widget. On (b) we add the new slot signature.

- Receiver combo box: select widget;

- Slot combo box: select the signal toggleBackgroundColor (bool) of widget.

As type-safe methods, the signatures of both sender and receiver methods must match. The parameter of toggleBackgroundColor() must be of the same type of toggled() - the bool type. It is also acceptable for a signal to have a signature with more arguments than a slot has. In this case the extra arguments are ignored [16].

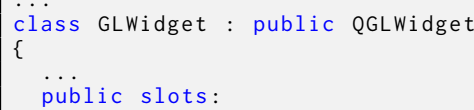




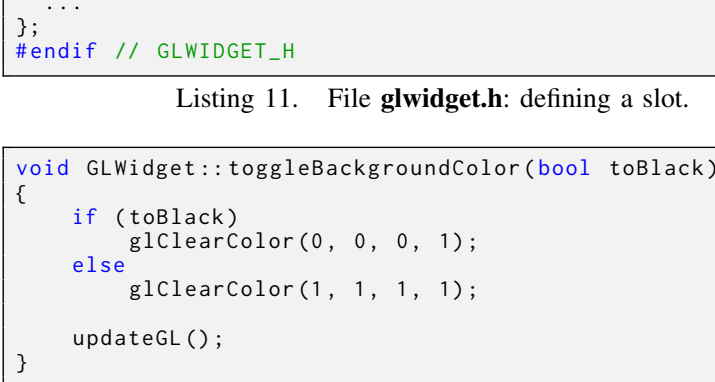

Listing 11. File glwidget.h: defining a slot.

Listing 12. File glwidget.cpp: implementing the slot.

Obs 1. Observe in Listing 12 the QGLWidget command updateGL(). We will use it whenever we need to repaint the widget.

\section{E. Loading a Geometric Model}

Our OpenGL application displays a triangular mesh model loaded from a file in Object File Format (OFF) format [17]. The user can choose the OFF file from a file selection dialog, accessed by File $\rightarrow$ Open on the menuBar.

To create such options File $\rightarrow$ Open, at Design mode click at Type Here on the menuBar and rename it to File. Qt Creator automatically creates the object menuFile of the class QMenu and opens a submenu. Click at Type Here on the submenu and change it to Open. Qt Creator automatically creates the object actionopen of the class QAction.

We want that, whenever the user selects Open at menu bar, the emitted signal, QAction: : triggered(), connects to a slot that displays a file selection dialog. We declare the slot as shown in Line 27 at Appendix A and define it as shown in Listing 13

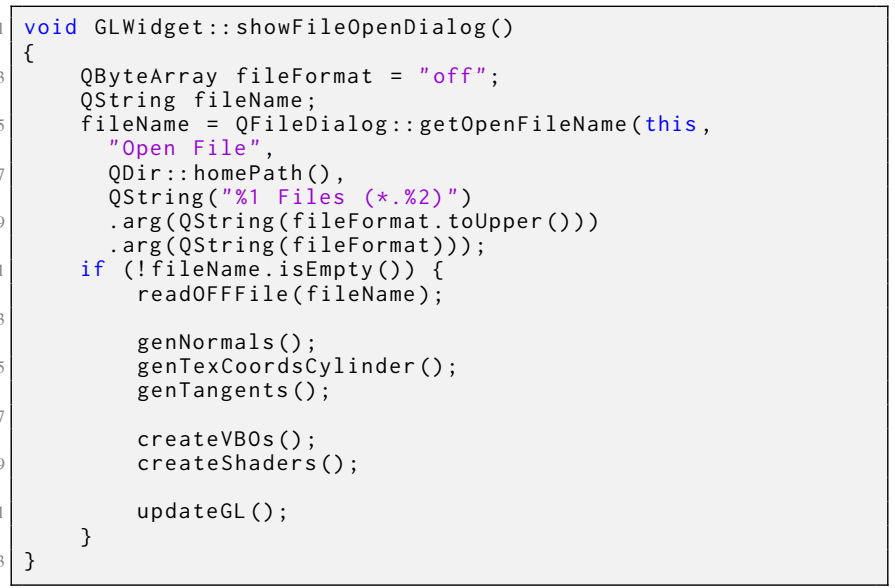

Listing 13. File glwidget.cpp: slot showFileOpenDialog().

Before detailing the new commands in the previous listing, let us conclude this signal/slot connection:

- Sender combo box: select actionopen;

- Signal combo box: select the signal triggered() of actionopen;

- Receiver combo box: select widget;

- Slot combo box: select the signal showfileOpenDialog() of widget.
In Listing 13, Line 5, the method QFileDialog::getOpenFileName() displays file open dialog box and returns the string containing the complete file path. At Line 12, the string is passed to the method readoffFile() (Listing 14), which reads the OFF file using the $\mathrm{C}++$ Standard Library class std::ifstream. In the array GLWidget::vertices, the mesh vertices are stored as QVector4D objects. The face indices are stored in the array GLWidget::indices as unsigned int values. The number of vertices and faces are stored in GLWidget::numVertices and GLWidget::numFaces, respectively. This method also centers and resizes the mesh.

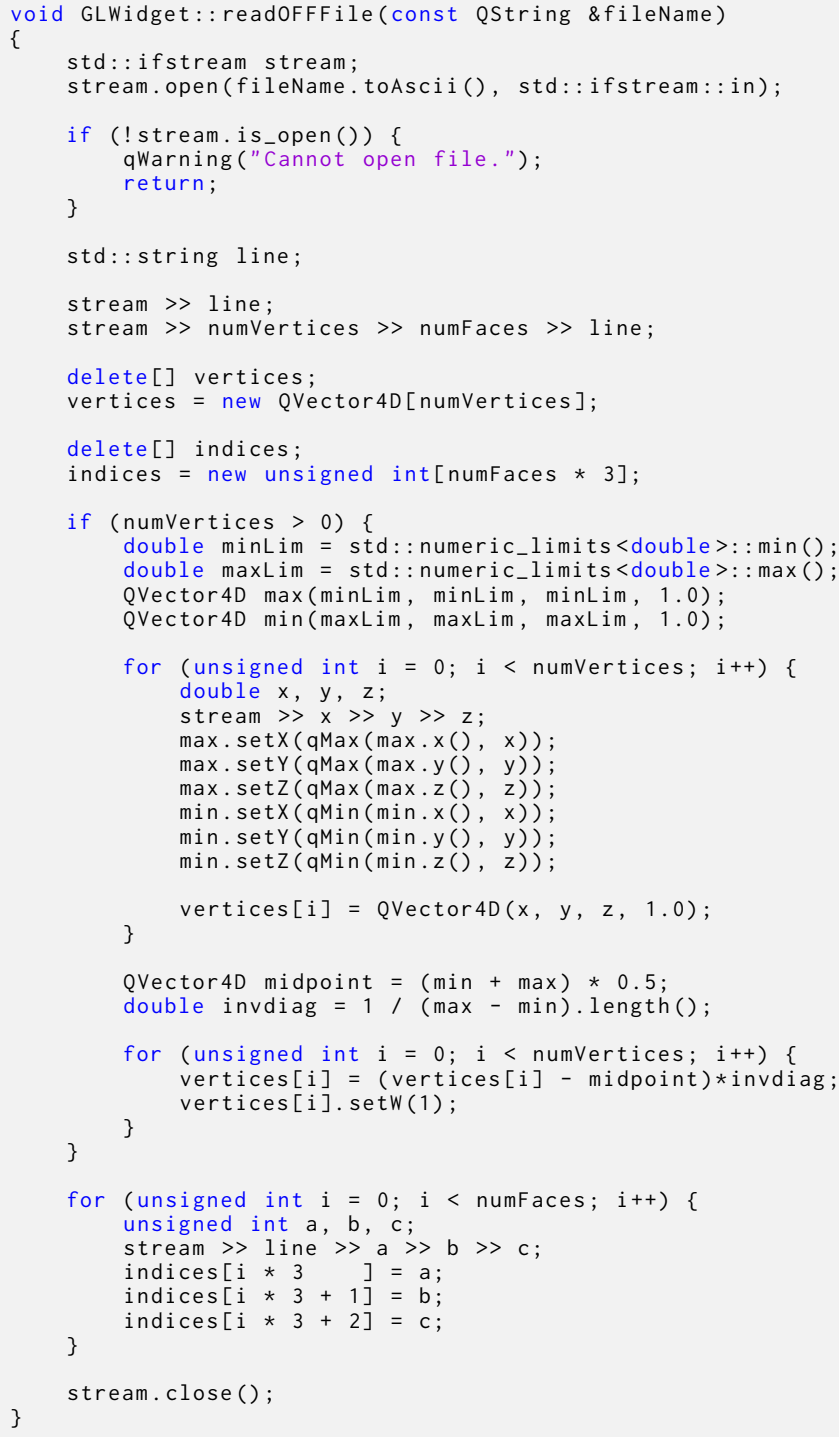

Listing 14. File glwidget.cpp: method readoffFile().

In Listing 13, Line 14, the method genNormal() (Listing 15) estimates the normals at the mesh vertices and stores them in the array GLWidget: : normals of QVector3D objects.

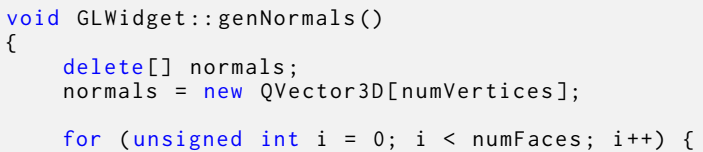




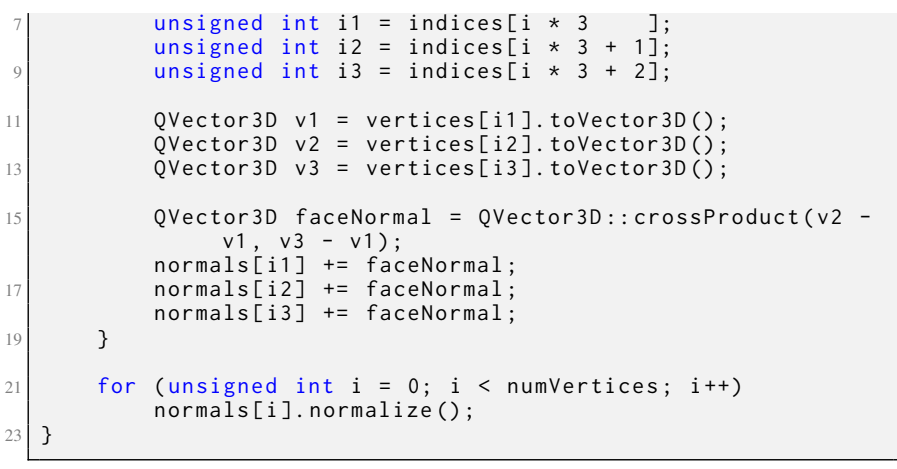

Listing 15. File glwidget.cpp: method genNormals().

In Listing 13, Line 15, the method genTexCoordsCylinder() (Listing 16) generates cylindrical texture coordinates for the mesh vertices. They are stored in the array GLWidget: : texCoords of Qvector2D objects.

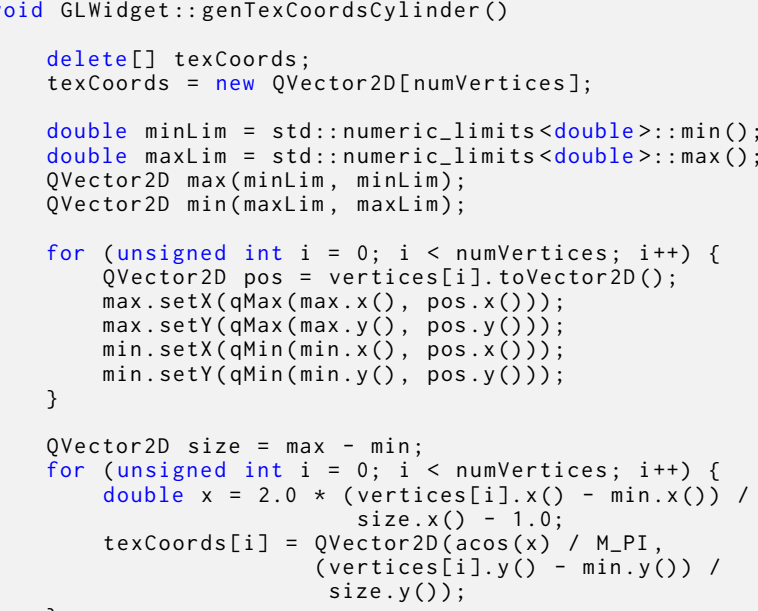

Listing 16. File glwidget.cpp: method genTexCoordsCylinder().

In Listing 13, Line 16, the method genTangents() (Listing 17) estimates per-vertex tangent vectors required by Normal Mapping [10]. Our code is based on the method described by Lengyel [18], [19]. The tangent vectors are stored in the array GLWidget: : tangents of QVector4D objects.

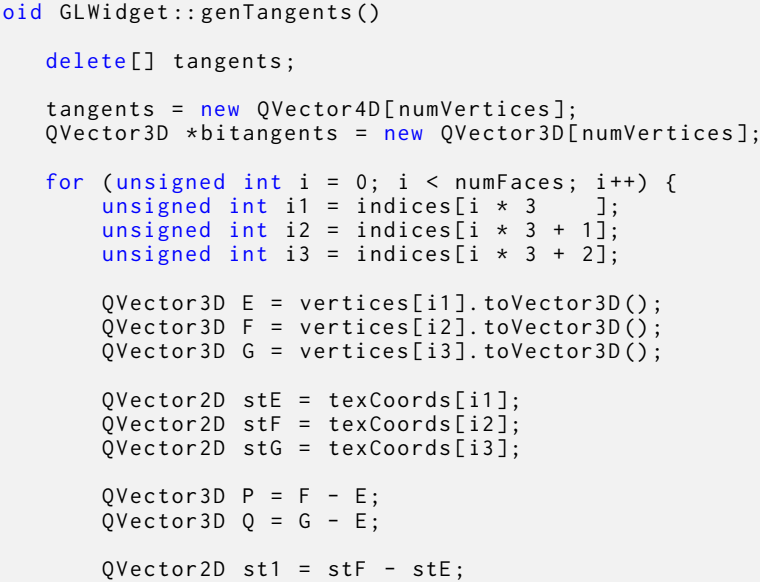

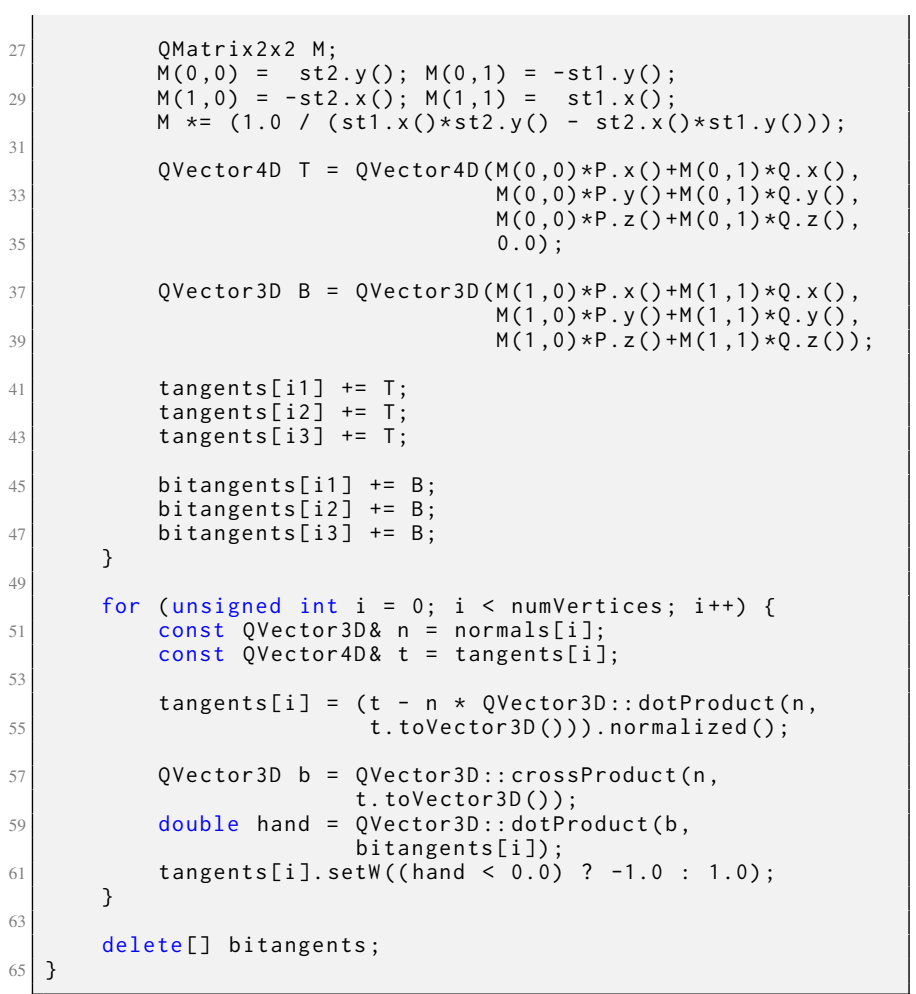

Listing 17. File glwidget.cpp: Method genTangents().

The remaining methods, createVBOs() and createShaders(), will be detailed in due course.

\section{F. Encapsulating Resources}

Graphics applications commonly make use of external asset files such as textures, icons, XML data and other text or binary files.

Qt provides a mechanism called Resource System [20] that stores external binary and text files into the application executable, encapsulating both application and their resources into a single binary file. This embedment of resource files is done during the build process. In our application, we will use this mechanism to store textures and GLSL programs.

In order to use the Resource System, we first add to our Qt project a resource collection file [20]. This is an XML file, with extension .qrc, that lists the resource files to be embedded. Again, we do not have to edit this XML file directly because Qt Creator provides a friendly interface, the Resource Editor, for managing resources (Fig. 12).

In Qt Creator, first select File $\rightarrow$ New File or Project then select Qt and Qt Resource file (Fig. 11-(a)). We name it resources.grc (Fig. 11.(b)).

Double click the file resources.qrc at Qt Creator to open the Resource Editor. Before we include the resource files, we must create a path prefix to organize the lists of files. We will create two prefixes, one for GLSL programs and other for texture files. Click at Add $\rightarrow$ Add Prefix and type /shaders. Repeat the process and type /textures. Now, to include resource files, click at the corresponding prefix and at Add $\rightarrow$ Add Files (Fig. 12). In Sections II-G and II-L we show how to access these resources. 


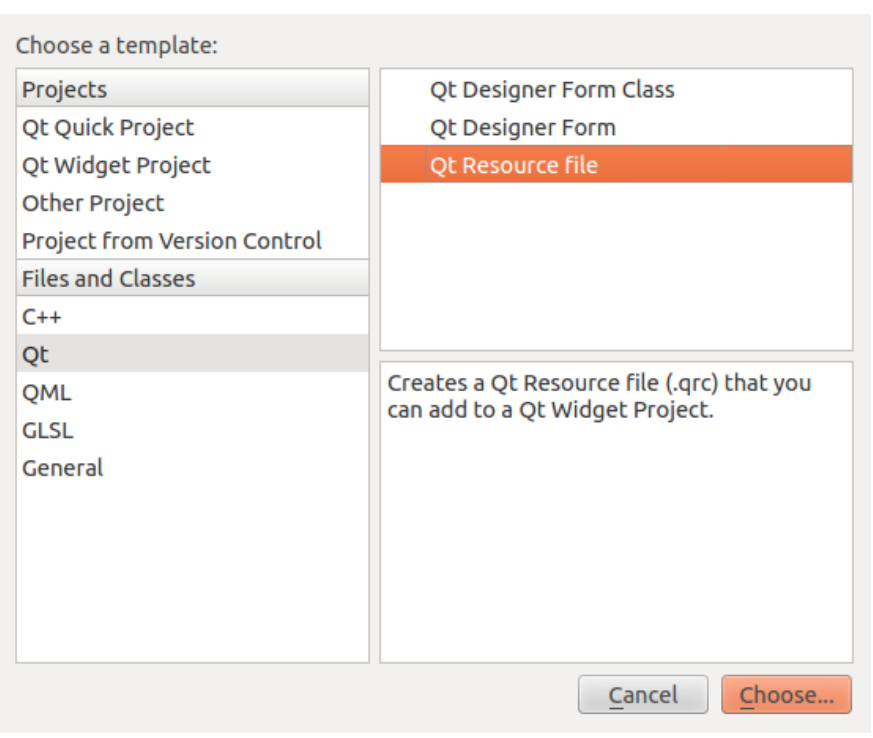

(a)
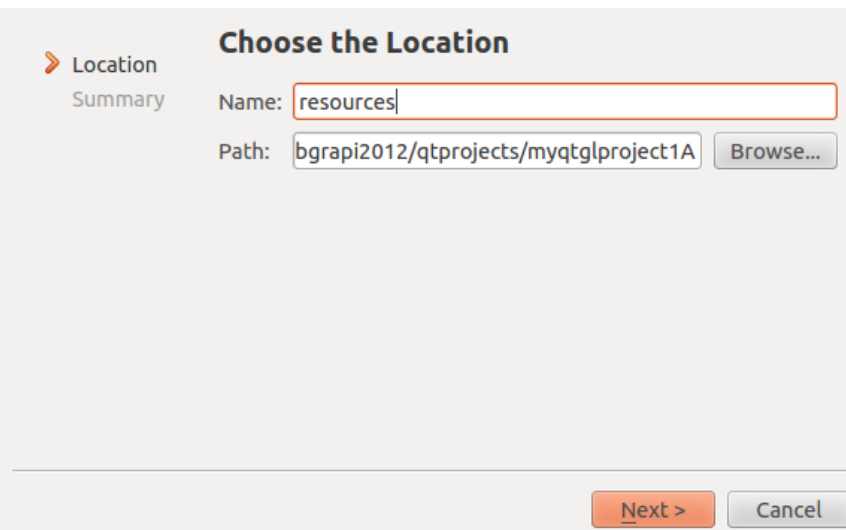

(b)

Fig. 11. Creating a Resource System using Qt Creator: on (a) we select the option Qt Resource file. On (b) we name our Resource System as resources.

Obs 2. Resource files embedded into the application executable are only accessible by Qt classes. For instance, if we want to open a text file managed by the Resource System, we cannot do that using std: :ifstream, instead we should use QFile [21].

Obs 3. If we try to run the application but Qt Creator produces an error message related to resource files, make sure to run Qmake by selecting, on the Qt Creator menu bar, Build $\rightarrow$ Run qmake before trying to run the application again.

\section{G. OpenGL Shaders and $Q t$}

Qt provides facilities to handle GLSL shaders and shader programs. Qt Creator features a built-in GLSL editor, while the QtOpenGL Module implements the classes QGLShader and QGLShaderProgram. The former class allows OpenGL shaders to be compiled and the later class allows OpenGL shader programs to be linked and bound to the OpenGL pipeline. In the Qt Creator, GLSL shaders can be included into the Qt project similarly as adding $\mathrm{C}++$ classes and resources.

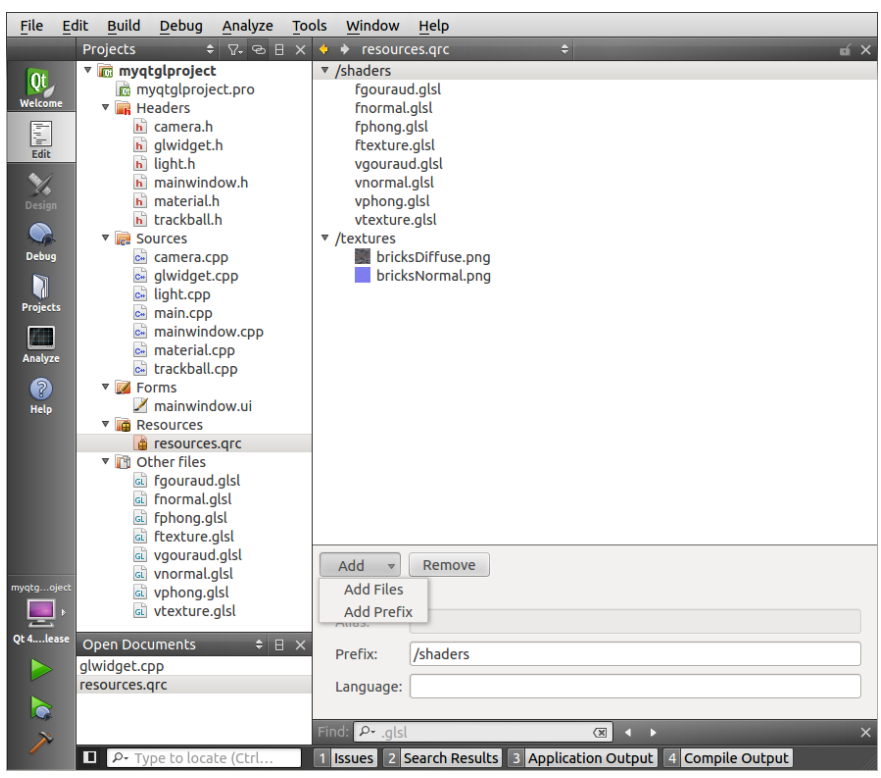

Fig. 12. Using the Resource System in our application: there are two prefixes, /shaders and /textures.

In our application, the user can use the keyboard to select among four shader effects that render a:

- Gouraud shaded model;

- Phong shaded model;

- Phong shaded model with texture mapping;

- Phong shaded model with normal mapping.

The source code of these shaders are listed in Appendix C

When the user selects a shader effect, the previously selected is firstly released from the OpenGL pipeline in order to the shaders of the current effect be compiled, linked and bound to the OpenGL application in run time. Details about compiling, linking and binding GLSL shaders are found in [8], [9], [10].

In Listing 18, we present our methods responsible to release and destroy the previous shader program and to compile and link the new one. The binding will be done in the paintGL() method (Sec. II-M. Listing 27).

It is worth to mention that we opt to use Qt Resource System to store our GLSL shaders. However, we could instead load them as text strings into the $\mathrm{C}++$ sources or from external files [8], [9], [10].

In order to load the shader files managed by the Qt Resource System to the application, their paths must begin with a colon followed by the resource prefix that we specified in the Resource Editor, i.e., :/shaders (Listing 18, Lines 5 and 11). On Lines 18 and 22 we create QGLShader objects for vertex and fragment shaders. On Lines 19 and 23 the corresponding GLSL source files stored in the Resource System are compiled. In our application, only the vertex and fragment shaders of the currently selected effect are compiled.

After compiling the shaders (using compileSourcefile()), we add (addShader()) them to the object shaderProgram of class QGLShaderProgram (Lines 27 and 28) and link (link()) 
the shader program (Line 30).

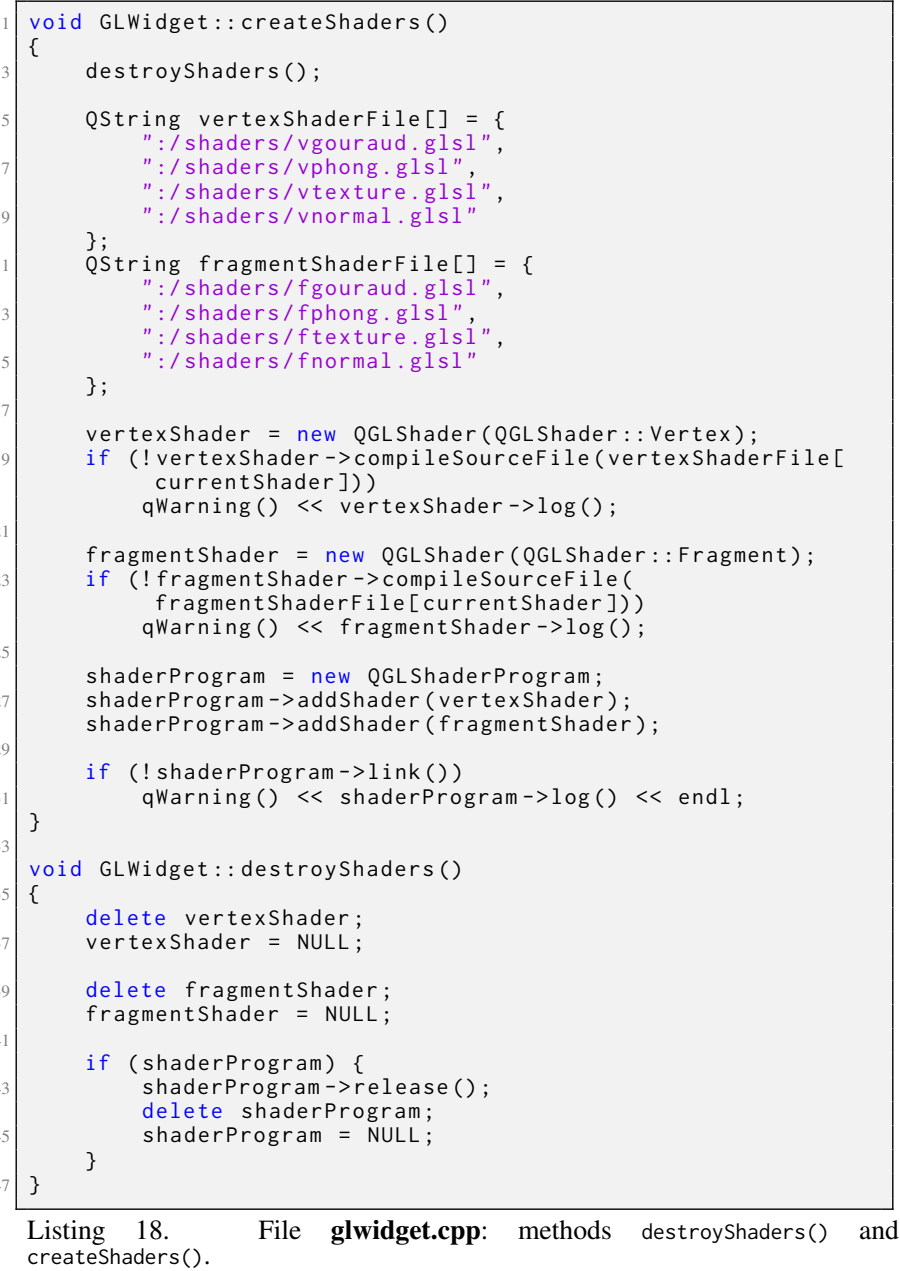

In Sec. [II-M, where the method paintGL() is detailed, we will present how to set up the input attributes and uniform variables of the shader program currently in use.

\section{H. Buffer Objects}

For a long time, the OpenGL's immediate mode and display lists [9] have been the standard approach to upload rendering data to the graphics server, but both features are now deprecated. Another option is to use OpenGL vertex arrays, but they are stored in the client side, which means that they must be sent to the server every time the scene is updated.

The concept of Vertex Buffer Objects (VBOs) [8], [22], introduced in OpenGL 1.5, allows the direct manipulation of the data stored in the server side. This is the approach used in our examples.

Qt provides the class QGLBuffer [23] to handle buffer objects. In our application we make use of four vertex buffers, according to Listing 19 .

- vboVertices: vertex positions of type QVector4D (Line 5);

- vboNormals: vertex normals of type QVector3D (Line 13);

- vboTexCoords: texture coordinates of type Qvector2D (Line 21);

- vboTangents: tangent vectors of type QVector4D (Line 29).

For each vertex buffer, we first create a buffer object using the parameter QGLBuffer::VertexBuffer in the constructor of QGLBuffer. We then create $(\operatorname{create}())$ the buffer in the server and associate (bind()) it to the current OpenGL context. Since our vertex data will be set only once and used many times for rendering, we set the QGLBuffer: :StaticDraw usage pattern at setUsagePattern(). Additional patterns can be found in the documentation [23].

We use the method allocate() to send the vertex data to the VBO as a contiguous untyped data. According to the specified usage pattern, OpenGL will decide where the data must be stored and when it must be sent [22]. Lastly, we can delete the array of vertex attributes passed to allocate() because it was already copied to the VBO.

We also create a buffer object to the indices of the mesh triangles (vboIndices, Lines 37-43). It only differs from vertex buffers, on the use of QGLBuffer: : IndexBuffer in the constructor.

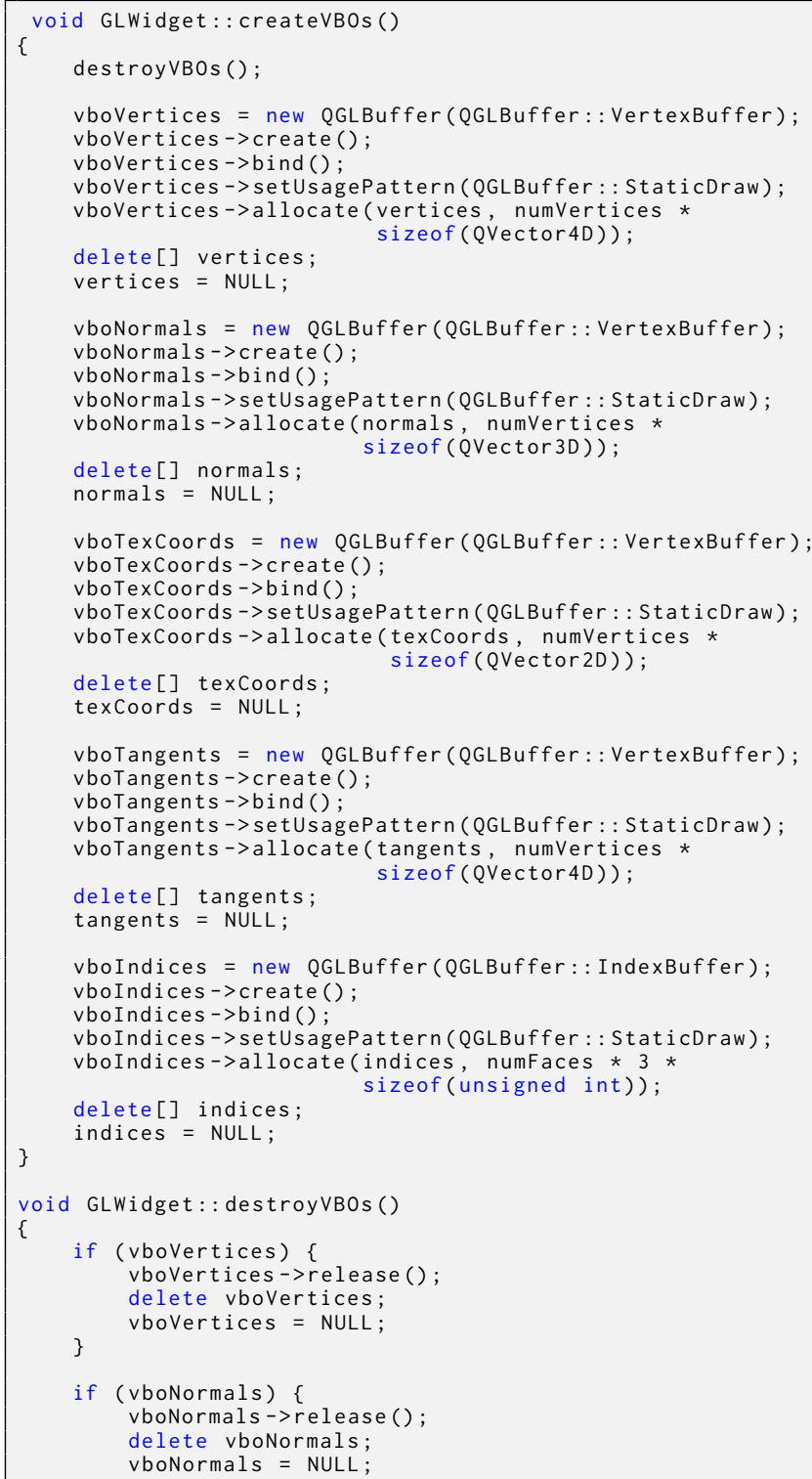




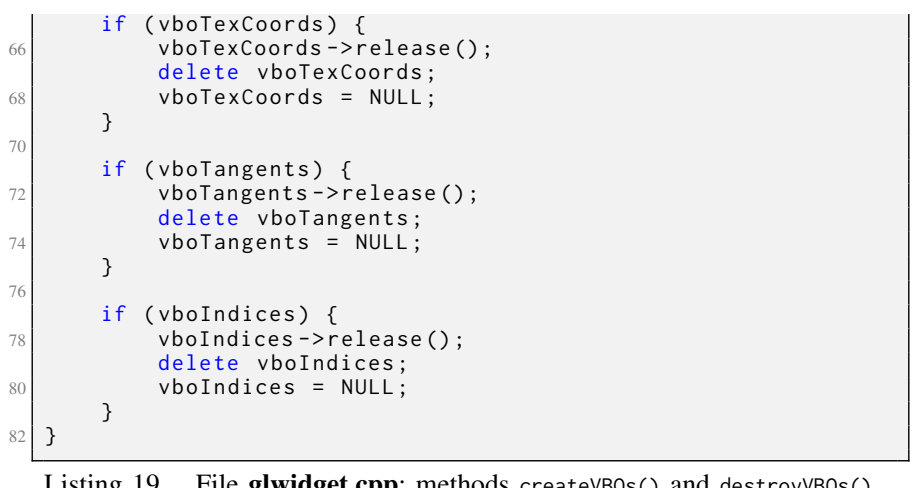

Listing 19. File glwidget.cpp: methods $\operatorname{createVBOs()}$ and destroyVBOs().

Obs 4. A key feature of the buffer objects is their capability to map their data into the client side. These allow us to efficiently update VBO data. In Listing 20 we show a simple example, where the methods map()/unmap() are employed to this purpose. Further options of buffer object mappings are found in [23].

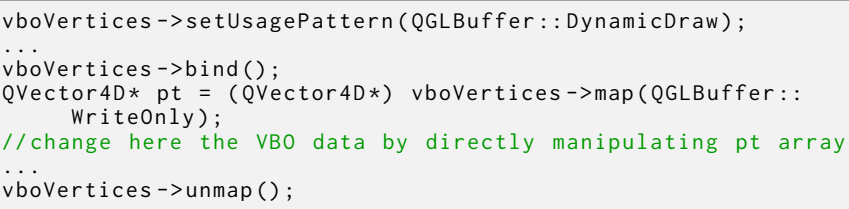

Listing 20. An example of mapping VBO data into client side.

\section{Mouse and Keyboard Events}

So far we learnt with signals and slots how to communicate between Qt objects. In this section, we will see that Qt also offers the capability to handle events from the window system [24]. In particular, we are interested in events generated by mouse and keyboard. Recalling GLUT, the mouse and keyboard events are handled by callback functions [2]. Qt, in turn, provides virtual methods in QGLWidget which are called in response to mouse or keyboard events.

In Listing 21 we present the method keyPressEvent() that handles keyboard events. This is the method responsible to switch among the shader effects (Sec. II-G) by pressing the keys 0 (Gouraud shading), 1 (Phong shading), 2 (Phong + texture) and 3 (normal mapping). Also, pressing the escape key, the application quits (qApp is a Qt global pointer to the unique application object).

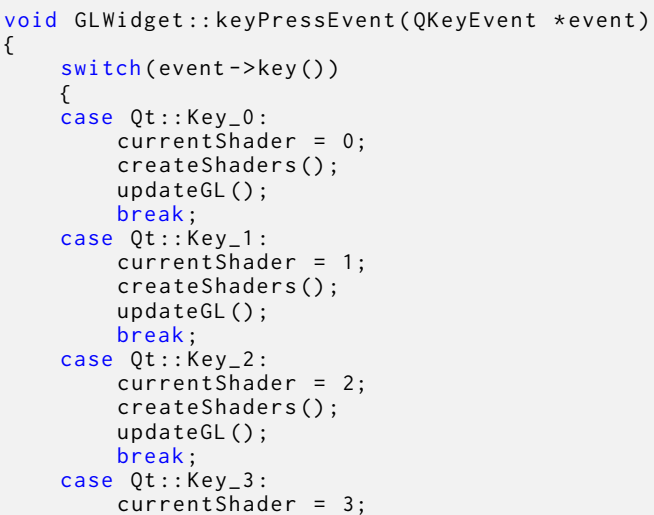

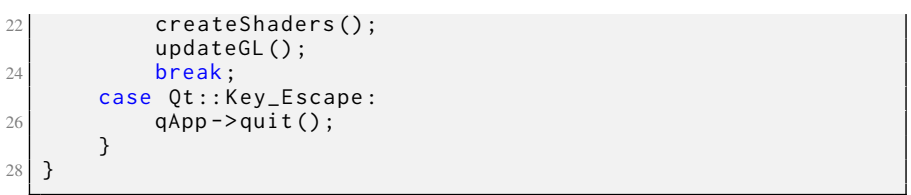

Listing 21. File glwidget.cpp: method keyPressEvent().

Obs 5. By default, widgets are set to not receive the keyboard or mouse focus. Therefore they will not catch any events from such input devices. In order to ensure that GLWidget will receive focus, we change (in Design mode) the focusPolicy property to a policy differing from the default $\mathbf{Q t :}$ :NoFocus. In our application, we use $\mathbf{Q t}:$ :StrongFocus, as it allows the widget to receive focus by both tabbing and clicking.

We also handle mouse events (Listing 22) for manipulating a virtual trackball. Appendix D presents the class TrackBall. This class implements the methods mouseMove(), mousePress() and mouseRelease() which are called in the event handlers in Listing 22. The only parameter of these trackball class methods is the current mouse position (event->posf()). For the wheelEvent() we simply implement a zooming operation, where event->delta( ) provides the distance that the mouse wheel is rotated (in eights of a degree).

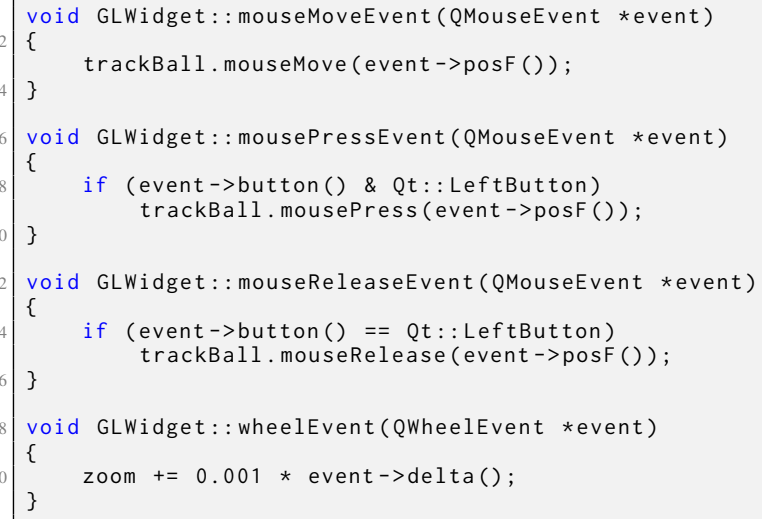

Listing 22. File glwidget.cpp: methods for mouse events used by the virtual trackball and for zooming.

\section{J. Matrix and Vector classes}

Qt provides a set of classes of vectors (Qvector2D, QVector3D, and QVector $4 \mathrm{D}$ ) and matrices (QMatri $\times 2 \times 2$, QMatri $\times 2 \times 3, \ldots$, QMatri $4 \times 3$, QMatri $\times 4 \times 4)$ to work with geometric transformations and camera settings. Their contents can be bound to shader attributes declared as native data types vec2, vec3, vec4, mat2, mat3, mat4 or shader arrays, using methods of the class QGLShaderProgram. This binding procedure is shown in Sec. III-M

In GLWidget, we declare two QMatrix4x4 objects, modelViewMatrix and projectionMatrix (App. A). In our application, they will replace the deprecated OpenGL matrix modes GL_MODELVIEW and GL_PROJECTION, respectively. However, we are now under the object-oriented paradigm, instead of the traditional state-machine OpenGL. 
In the method resizeGL(), in Listing 23, we set up projectionMatrix. First we initialize the projection matrix to the identity matrix (equivalent to a call of glLoadIdentity() under gIMatrixMode(GL_PROJECTION)). We then right-multiply itself by the matrix corresponding to the perspective projection, using the method QMatri $4 \times 4$ : : perspective(). This method has the same parameters of gluPerspective().

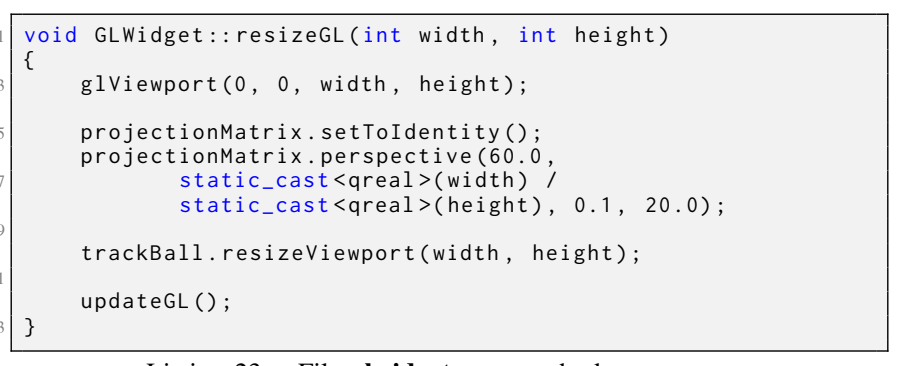

Listing 23. File glwidget.cpp: method resizeGL().

In the method paintGL() (Listing 27), we set up modelViewMatrix. As projectionMatrix, we first set it to the identity matrix. After that, we use the method QMatrix4×4: :lookAt() which, similarly to gluLookAt(), creates a viewing matrix derived from the triple: a observer point, a look-at point and an up-vector. We also apply two geometric transformations: first a rotation, provided by the trackball object (App. D), and second a translation. Notice that, as the deprecated OpenGL geometric transformations (glTranslate/glRotate/glScale), Qt matrix transformations correspond to right-multiplications.

Obs 6. QMatri $\times 4 \times 4$ provides other matrix transformation methods, e.g., projection matrices frustum(), ortho() and the geometric transformation scale(). Overloaded operators for matrix-matrix and matrix-vector operations are also provided. Further methods as well as their details are found in the QMatri $\times 4 \times 4$ documentation [25].

\section{K. Signals/Slots - Part 2}

So far we learnt how to work with signals/slots using Qt Creator. In this section we show other objects, not directly related to UI, that are also able to intercommunicate using signals/slots.

We exemplify with an instance of the class QTimer, timer, declared in GLWidget. It emits signals to widget in order to repaint the OpenGL window during the application's idle time.

Instead of defining the signal/slot connection in Design mode, we do that directly in the source code. In Listing 26. Line 12, the Qt command connect() connects the pre-defined signal timeout() emitted by timer to the custom slot animate() (Listing 24) of the widget. In Line 13 of Listing 26, we start the timer with a timeout interval of zero milliseconds, which means that a timeout signal will be emitted whenever the application is idle.

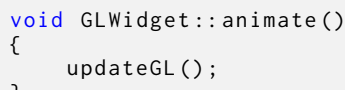

Listing 24. File glwidget.cpp: slot method animate().
We also exemplify a custom signal defined in our class GLWidget that connects to a pre-defined slot. This signal will be connected to a slot of the status bar that shows on it the number of vertices and faces of the loaded mesh. When the mesh model is successfully loaded in readOFfFile() (Listing 14], widget emits the statusBarMessage() signal. This is done by inserting Lines 4-6 to the end of readoffFile(), as shown in Listing 25. We create the connection in Design mode, associating our custom signal statusBarMessage() from widget to the pre-defined slot showMessage() of statusBar.

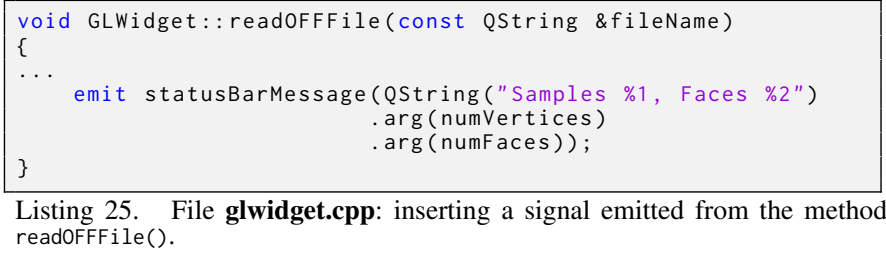

Listing 25. File glwidget.cpp: inserting a signal emitted from the method readOFFFile().

Obs 7. Signals do not need to be defined, only declared: in this last example we do not define the method statusBarMessage(); it is only declared in GLWidget (Listing 30 . Lines 22 and 23) using the reserved $Q t$ keyword signals.

\section{Texture Mapping}

We will use two texture maps: a diffuse map and a normal map [8]. In Listing 26, Lines 5-6, we create the objects texColor and texNormal of class QImage by loading image files from the resource collection file. After that, in Lines 7-10 we generate corresponding 2D GL textures for the first two texture units (GL_TEXTUREO and GL_TEXTURE1). The method QGLWidget: : bindTexture() calls the OpenGL command glGenTextures(), binds the new texture and returns the texture identifier for future usage.

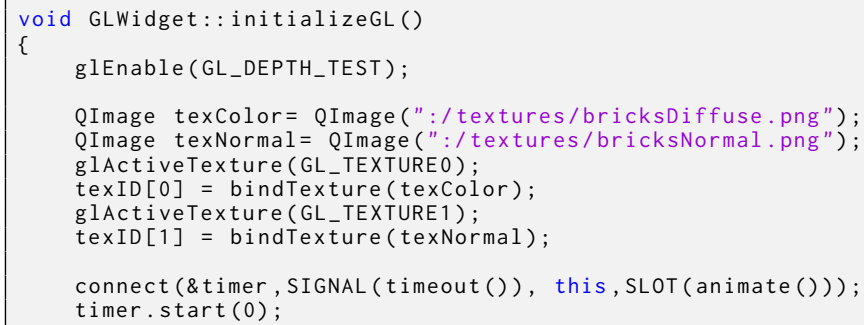

Listing 26. File glwidget.cpp: method initializeGL().

Obs 8. The concept of pixel buffer objects is another option to work with textures in OpenGL. The class QGLBuffer supports pixel buffer objects by using the Qt buffer types QGLBuffer: :PixelPackBuffer and QGLBuffer: :PixelUnpackBuffer. One of the advantages of pixel buffer objects is the asynchronous communication between the CPU and the GPU, which is useful, for instance, in applications where the textures need to be changed in run time [22].

\section{The method paintGL()}

The method paintGL() (Listing 27) is called whenever the OpenGL scene must be redisplayed. For each call, paintGL() performs the following tasks: 
1) The model-view transformations;

2) The binding of the shader program;

3) The uploading of the uniform data to the GPU;

4) The binding of the buffer objects and textures to the GPU;

5) The releasing of the buffer objects and the shader program.

The model-view transformations (Lines 8-11) were aforementioned in Sec. II-J. After binding the shader program (Line 13), we upload the matrices modelViewMatrix and projectionMatrix to the mat4 uniform shader variables modelViewMatrix and projectionMatrix (see the vertex shaders in App. C). We also upload the transpose of the inverse of the top-left $3 \times 3$ part of modelViewMatrix to the mat3 uniform variable normaMatrix (Line 17). This matrix is used to apply the model-view transformation to the vertex normals. In Lines 19-28, we upload the coefficients of the Phong lighting model (we assume only one light source).

The texturing code is shown in Lines 33-37. First we assign the sampler shader variables texColorMap and texNormalMap to the first two texture units (Lines 33-34). We see in the fragment shader ftexture.glsl (Listings 36 the use of texColorMap, whereas in the fragment shader fnormal.glsl (Listing 38) the use of both texColorMap and texNormalMap. Finally, in Lines 3336 we bind both textures to the corresponding texture units. Here we use the native OpenGL command glBindTexture(), passing as parameters the texture identifiers that we kept from the last call to QGLWidget: :bindTexture() when the textures were created (as shown in Sec. II-L, Listing 26 .

In Lines 38-53 we bind the different buffer objects and assign them to the corresponding shader program attributes. For instance, in Lines 38-40, vbovertices is bound. shaderProgram enables the array attribute vPosition (see its declaration on the vertex shaders in App. C) and finally, the method QGLShaderProgram: :setAttributeBuffer() formats vPosition, where its parameters are: the type of elements in the vertex array, the starting position in the bound buffer object, the number of per vertex components and the stride between consecutive vertices.

Just before calling the native OpenGL command glDrawElements() to draw the mesh, we bind the index buffer in Line 54.

The method paintGL () ends with the releasing of all buffer objects as well as of the shader program.

Obs 9. In our application, the uploading of shader variables and the binding of shader attributes in paintGL() is done irrespective of the shader effect currently set. This is acceptable because any variables and attributes not referenced in the shader program are simply ignored. For instance, the shader program for rendering a non-textured model with Phong shading does not require texture coordinates or tangent vectors passed as attributes. In such case, the binding of the corresponding buffer objects and textures are ignored.

Obs 10. The set up of shader attributes could be done outside paintGL() just after the model is loaded and the shader program is created, assuming that our application is composed of only one mesh model rendered with only one shader effect. Also, since the contents of our textures do not change along the execution of our application, we could perform the texturing work only once. However, we opt to leave these commands at paintGL() as it is done in more sophisticate applications with several mesh models, shader effects and textures.

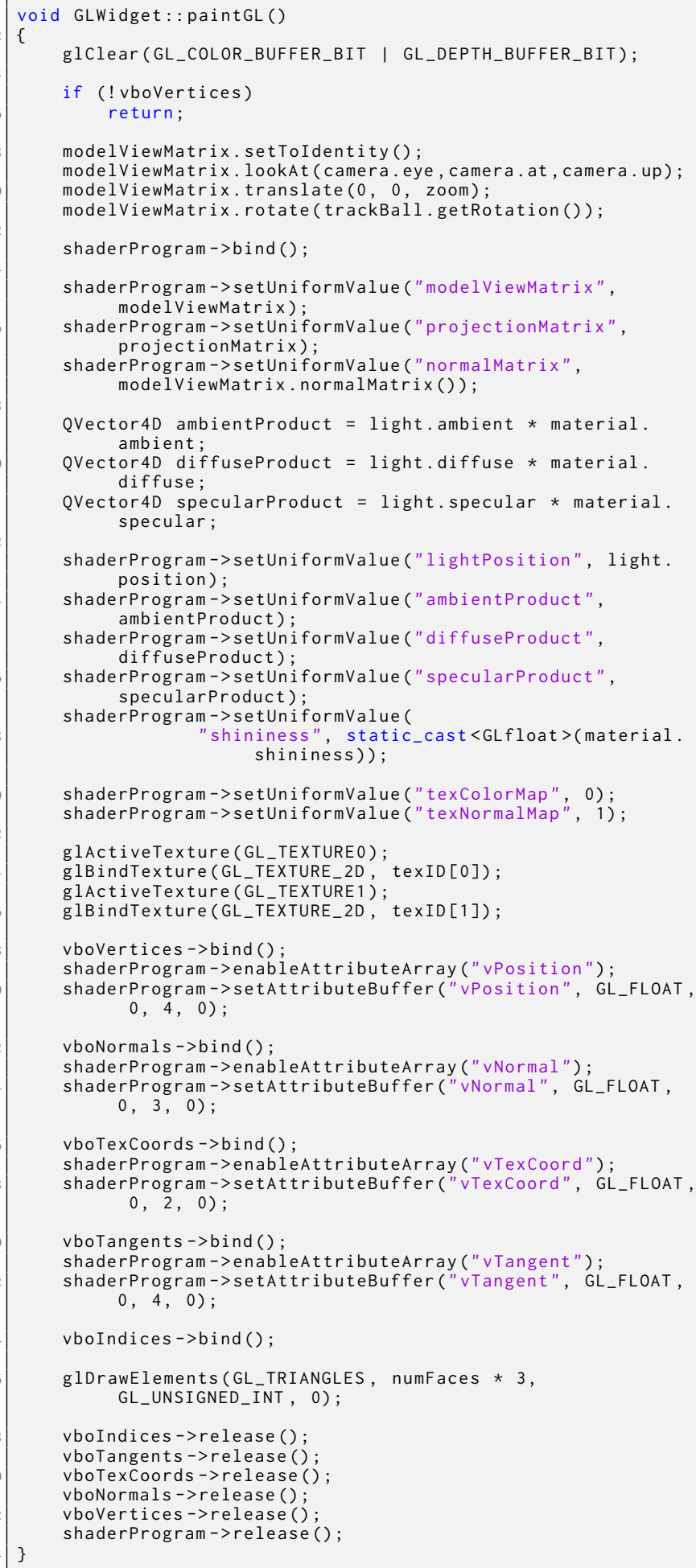




\section{N. Formatting the OpenGL context}

Qt provides the class QGLFormat [26] that allows to specify the display format of an OpenGL context. For instance, it allows to set double or single buffering (double buffering by default), alpha channel (disabled by default), stereo buffers (disabled by default), the color mode (rgba by default), the depth buffer (enabled by default) and antialiasing (disabled by default). The functionalities of this class are similar to those of the function glutInitDisplayMode().

We must format the OpenGL context prior to the creation of GLWidget. In our application, we first create a QGLFormat object format based on the default Qt format (Listing 28, Line 7) and enable the support to antialiasing (Lines 8-9). After that we set format to the new default OpenGL context format (Line 12). As can be noticed, this process is done in the main() function before the creation of the MainWindow object. When the MainWindow object is defined, our GLWidget automatically creates an OpenGL context using the new default format.

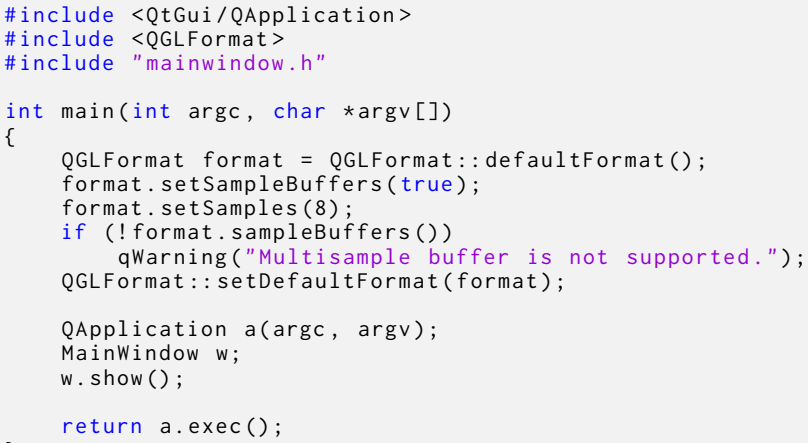

Listing 28. File main.cpp: setting the default format for the OpenGL context.

\section{IMPROVING THE USER INTERFACE}

So far the UI of our application is quite simple, as shown in Fig. 13 Qt provides several other UI components to improve the graphical interface of our application [4], [5]. In Fig. 14 we present a richer GUI with several tabs containing different sets of components. On (a) we can select the shader either by keyboard or by a combo box. On (b) we can change the colors of the light and the materials in the Phong lighting model by sliding dials. On (c) we can take a screenshot of the OpenGL scene by pressing a push button (see App. F for the source code of the slot associated to this button), we can set the background color using a QColorDialog object, as shown on (d), and we can toggle a check box to display the frame rate as an overlaid text string rendered by the method QGLWidget: : renderText().

\section{Final Considerations}

In this work we present how to develop an interactive graphics application with the Qt framework, OpenGL and GLSL using the Qt SDK. We show how such an application is built with reasonably small effort while keeping coding effectiveness. In our exposition we exploit programmable

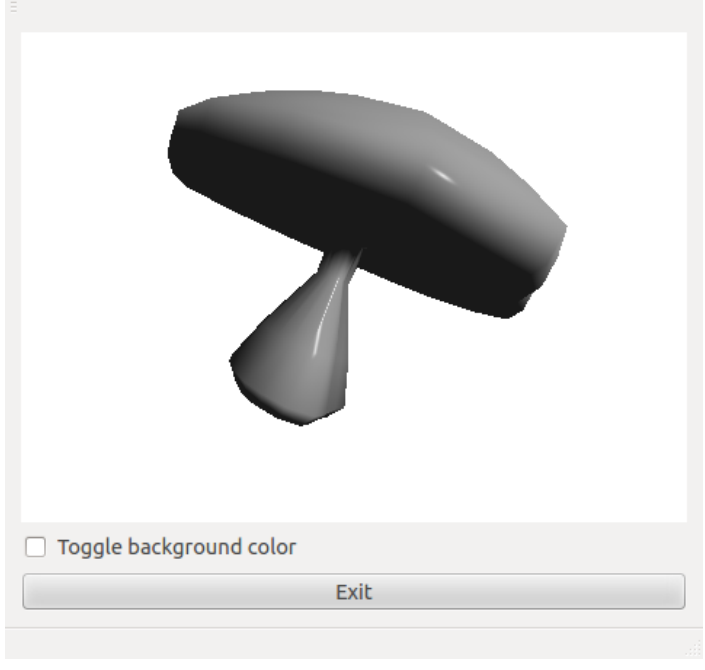

Fig. 13. Simple Qt with OpenGL application.

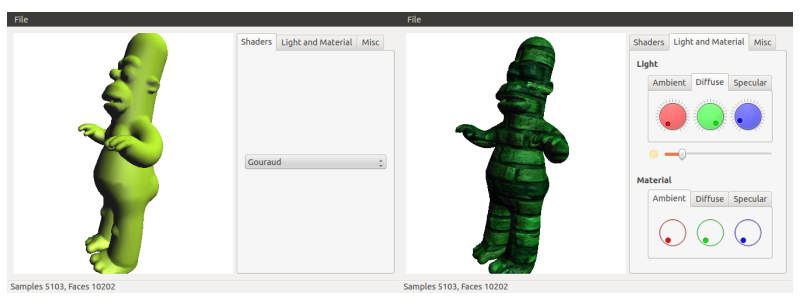

(a)

(b)

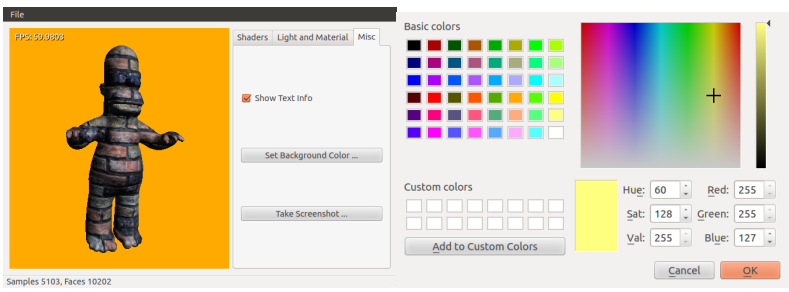

(c)

(d)

Fig. 14. An improved Qt application with OpenGL: several tabs and parameters can be tuned by the UI.

OpenGL pipeline features with Qt, e.g., buffer objects and shader programs. In particular, we do not use OpenGL 3.0 deprecated functions. We employ the Qt object-oriented program framework, allowing to encapsulate the newest OpenGL functionalities while maintaining the source code clean and organized.

We believe that OpenGL with Qt is a reasonable choice not only to develop academic and professional applications, but also to educational purposes as we have successfully applied this combination on both graduate and undergraduate computer graphics courses.

\section{Directions For Further Graphics-BAsed QT APPLICATIONS}

The power of Qt for interactive graphics applications is not limited to the scope of this presentation which relied on the 
Qt OpenGL Module.

For instance, for 2D graphics applications, Qt provides the classes QGraphicsScene and QPainter, which allow to draw and organize complex 2D vector elements, text fonts and pixmaps [4]. Furthermore, QPainter can be combined with QGLWidget to render scenes with 2D and 3D graphics elements (see, for instance, Qt Boxes Demo [27]).

Qt framework 5.0 will be released soon. This new major release will feature the Qt3D Module [28], which implements a scene graph using the Qt Meta Language QML. The scene can be described by nodes that correspond to geometry, material, effects and transformations.

\section{Acknowledgments}

The authors gratefully thank professor Siang Wun Song for his valuable comments on this work. The authors also thank professor Marcelo Walter, chair of SIBGRAPI's Tutorial 2012.

\section{APPENDIX A}

GLWidget CLASS DECLARATION

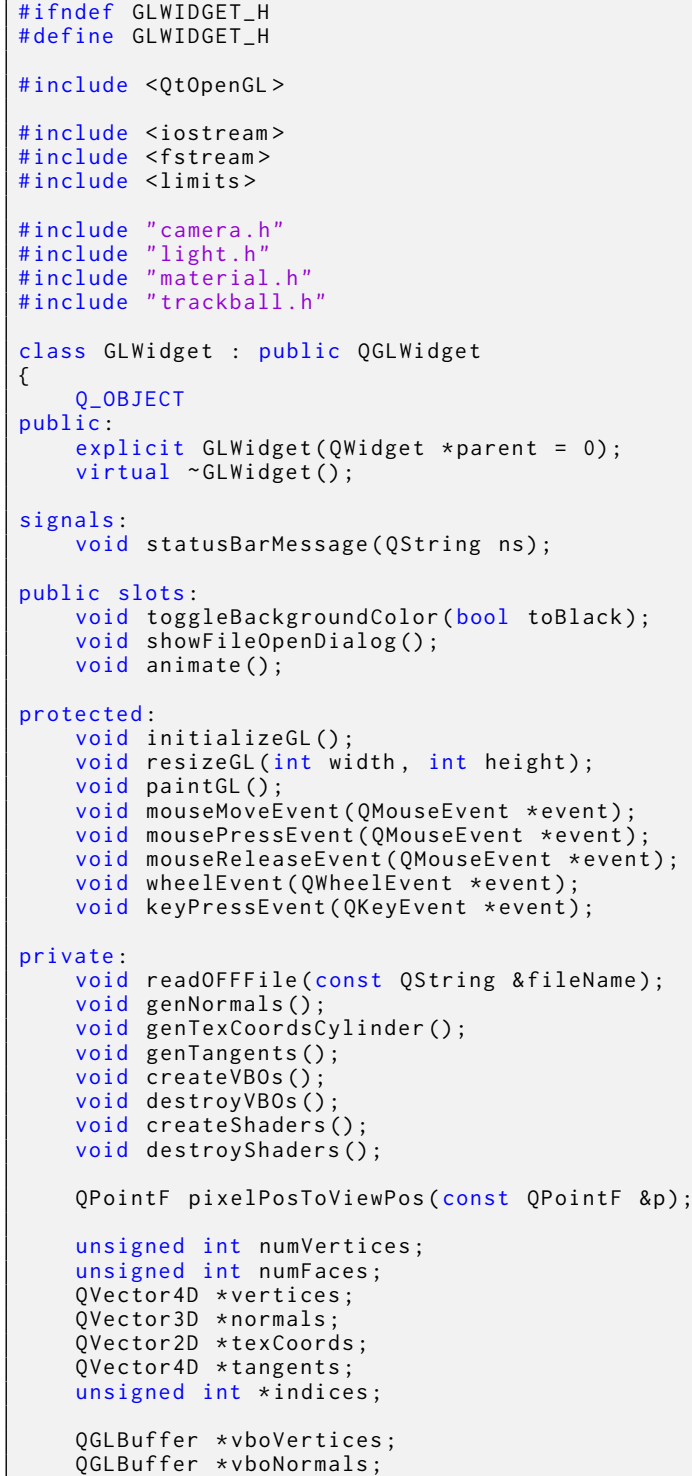

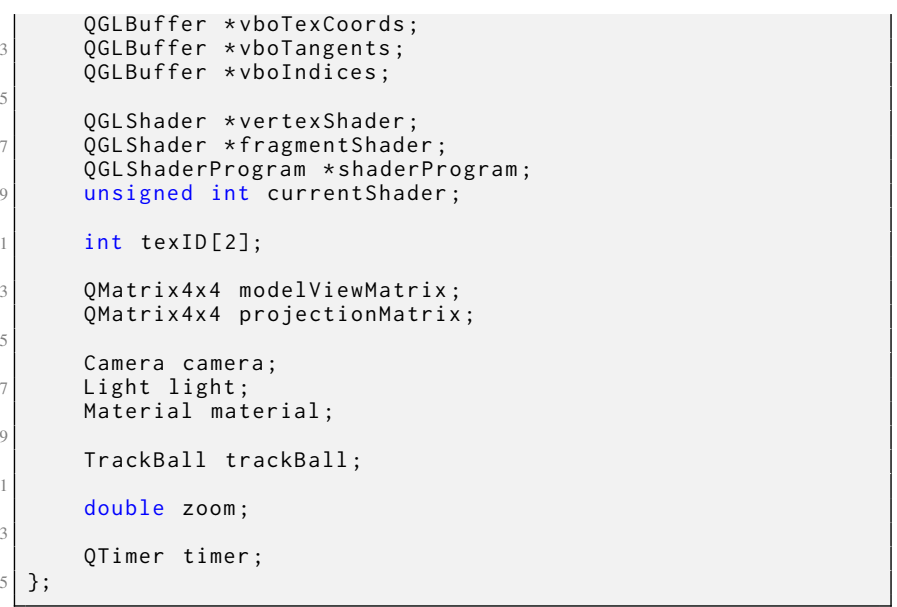

Listing 29. Class GLWidget.

\section{APPENDIX B}

\section{CONSTRUCTOR AND DESTRUCTOR OF GLWIDGET}

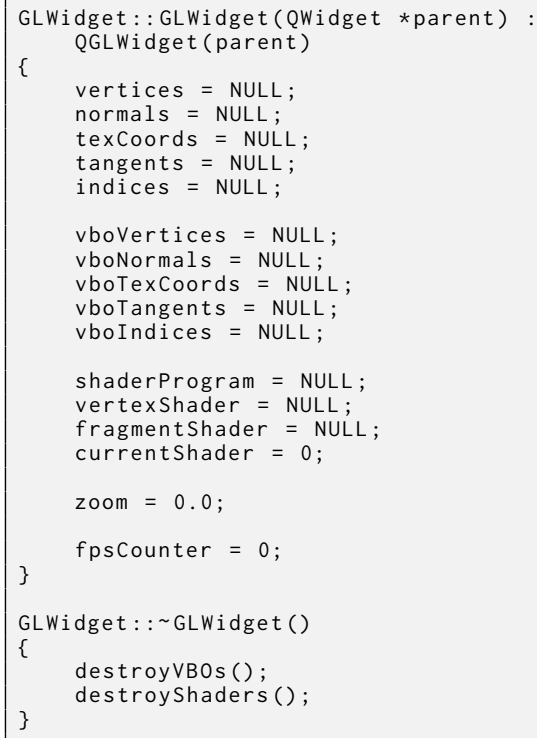

Listing 30. File glwidget.cpp: constructor and destructor of GLWidget.

\section{APPENDIX C GLSL SHADERS}

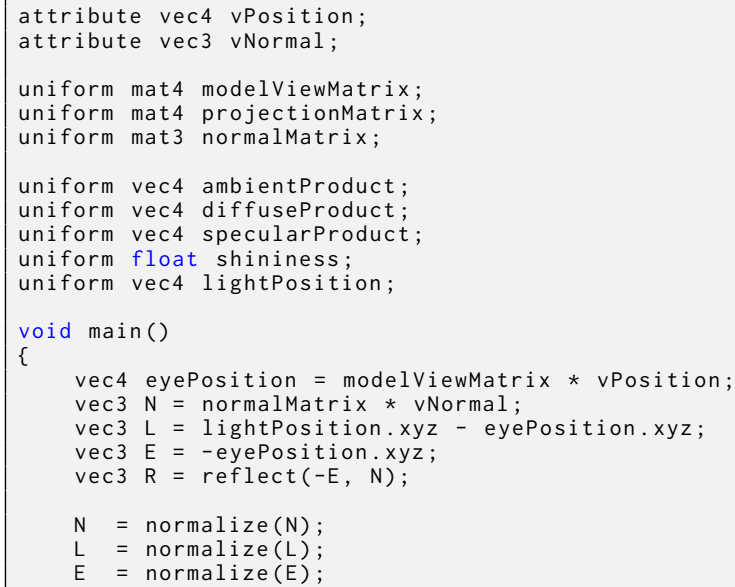




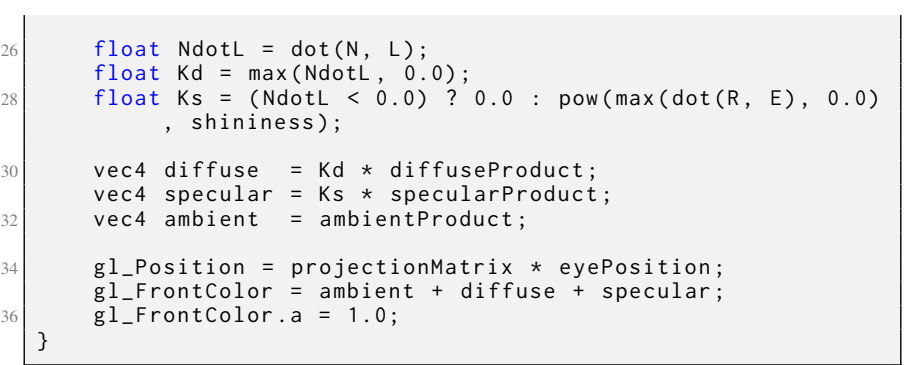

Listing 31. File vgouraud.glsl.

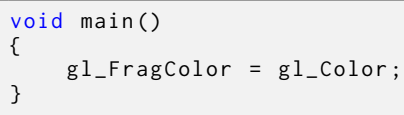

Listing 32. File fgouraud.glsl.

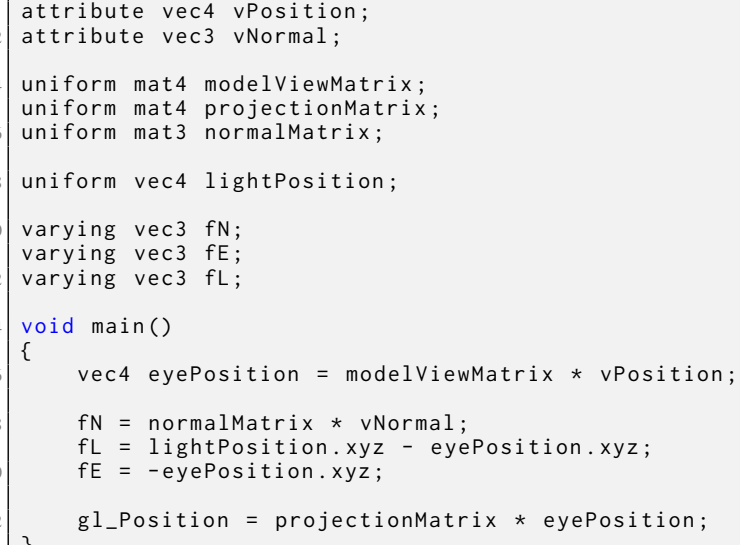

Listing 33. File vphong.glsl.

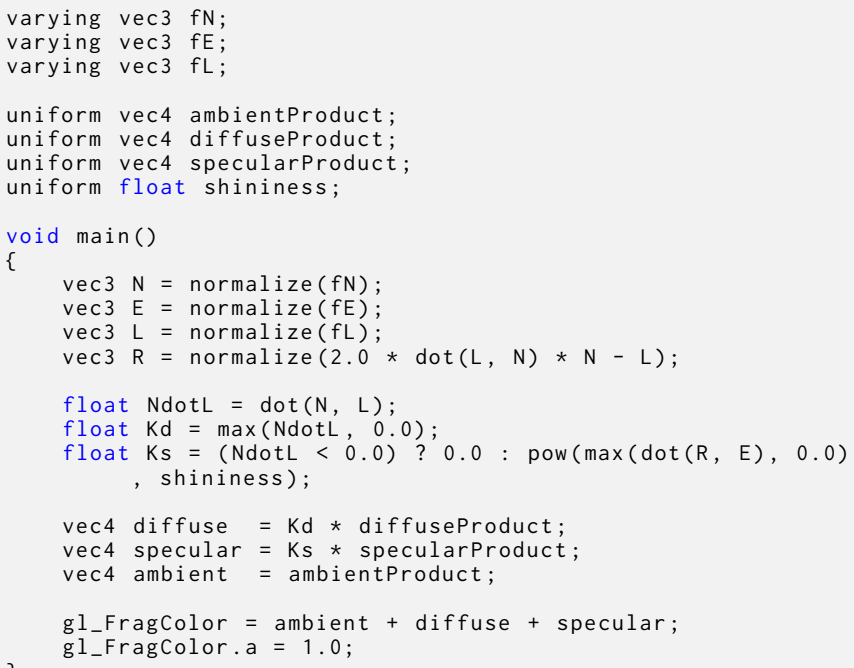

Listing 34. File fphong.glsl.

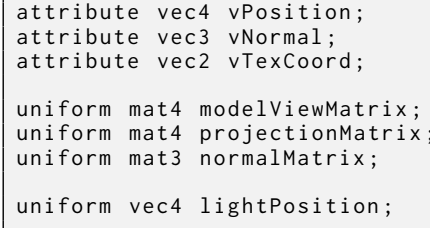

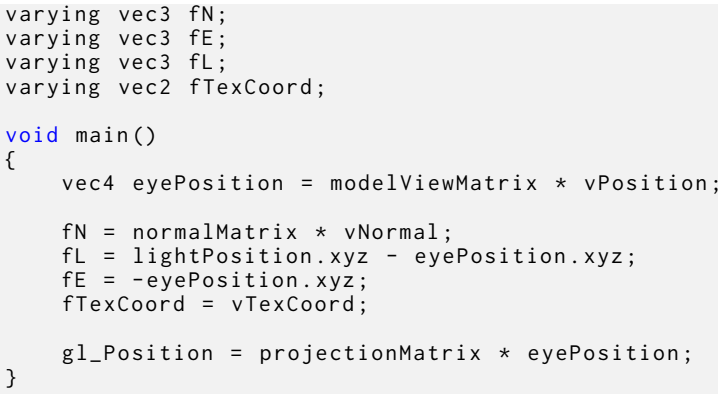

Listing 35. File vtexture.glsl.

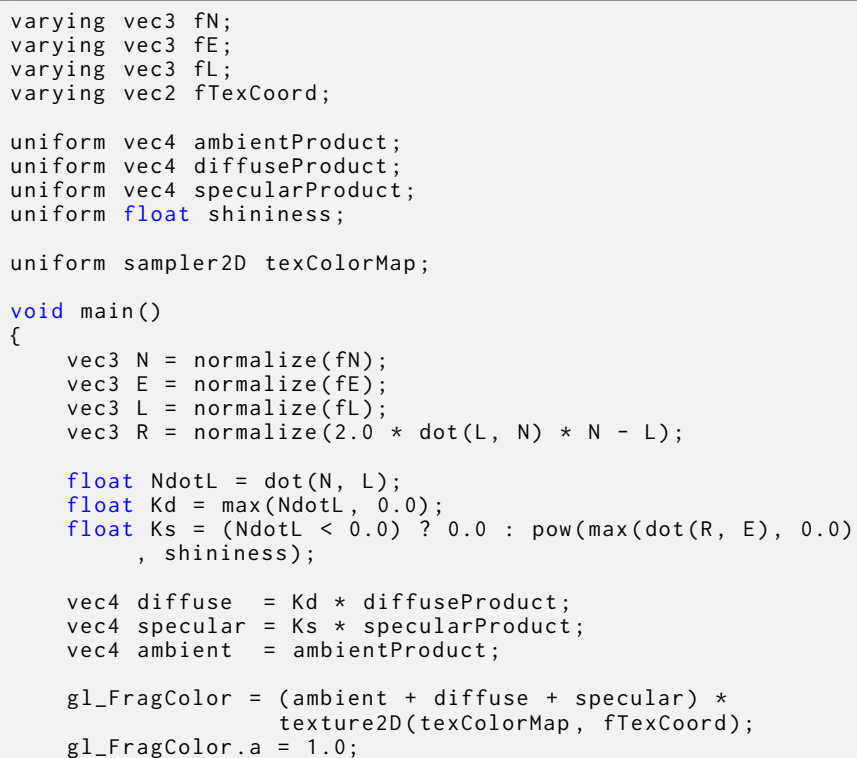

Listing 36. File ftexture.glsl

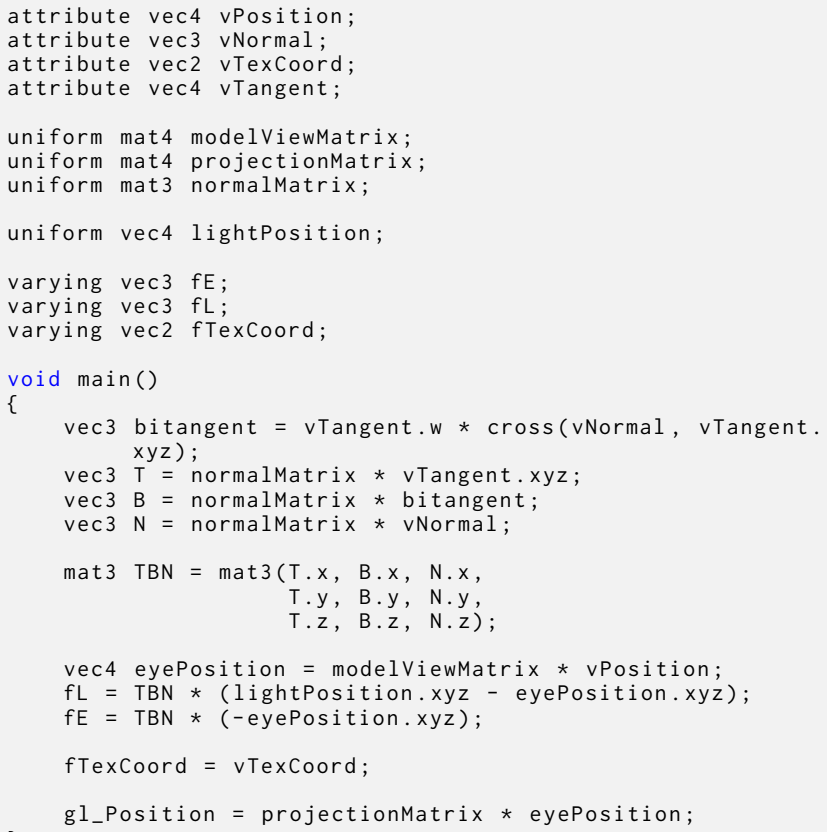

Listing 37. File vnormal.glsl. 


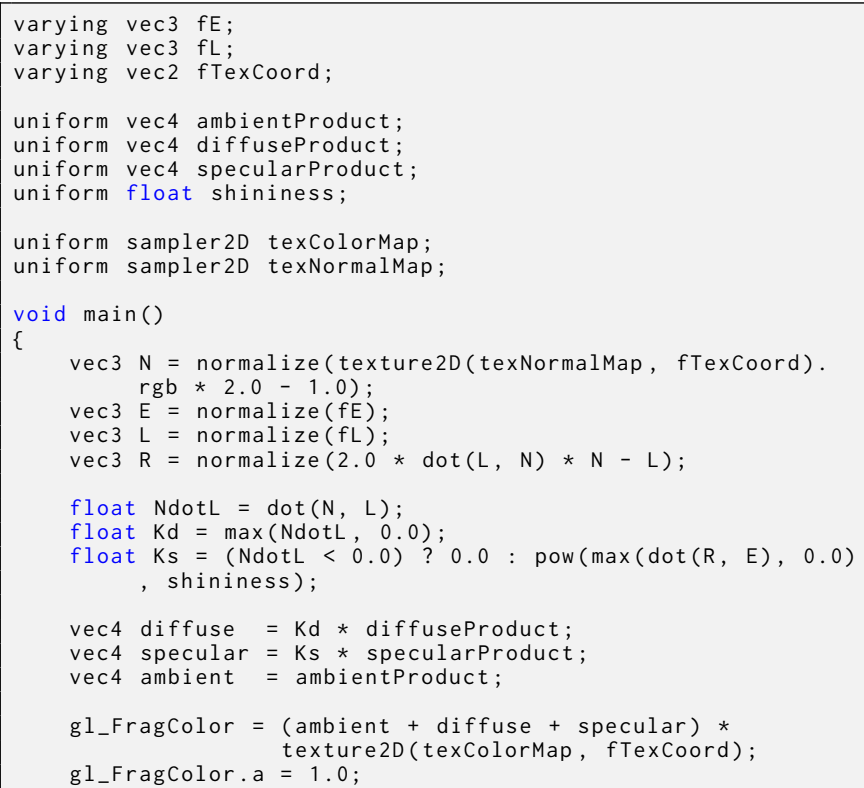

Listing 38. File fnormal.glsl.

\section{APPENDIX D}

\section{Virtual Trackball Class}

Our implementation of the virtual trackball was based on both the book by Angel and Shreiner [8] and on the Boxes Demo available on Qt framework 4.8 [27]. We use the class QQuaternion to represent rotations in 3D space using quaternions.

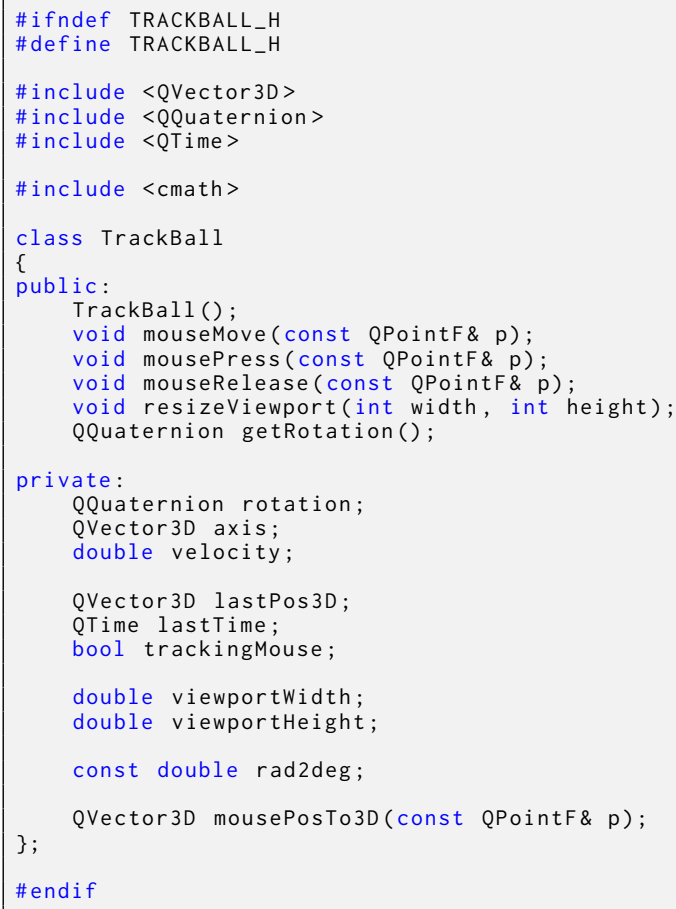

velocity $=0.0$

trackingMouse $=$ false:

void TrackBall: : mouseMove (const QPointF \&p)

if (! trackingMouse)

return;

QTime currentTime $=$ OTime: : currentTime ()$;$

int msecs = lastTime.msecsTo (currentTime);

if $(\mathrm{msecs})$ (

QVector3D vp = mousePosTo3D $(p)$.

QVector3D currentPos3D = QVector $3 D(v p . x(), v p \cdot y()$, $0.0)$;

double lensqr $=$ currentPos 3D. lengthSquared () ;

(lensqr $>=1.0)$ ? currentPos3D.normalize():

currentPos3D. setZ( sqrt (1.0 lensqr));

axis = QVector 3D: : crossProduct(lastPos3D, currentPos 3D):

double angle $=$ rad2deg * axis. length ();

velocity = angle / msecs;

axis.normalize ();

rotation = QQuaternion: : fromAxisAndAngle(axis, angle

$$
\text { ) * rotation; }
$$

lastPos3D = currentPos 3D;

\}

\}

void TrackBall: : mousePress (const QPointF \&p)

rotation = getRotation ();

trackingMouse $=$ true :

lastTime = QTime: : currentTime();

lastPos3D = mousePosTo3D $(p)$;

double lenSqr = lastPos $3 D$. lengthsquared ();

(lenSqr $>=1.0)$ ? lastPos3D.normalize ()

lastPos3D. setz(sqrt( 1.0 - lenSqr));

velocity $=0.0$

\}

void TrackBall: : mouseRelease (const QPointF \&p)

\{ mouseMove $(p)$;

\}

trackingMouse $=$ false;

void TrackBall: : resizeViewport (int width, int height)

\{

viewportwidth = static_cast $<$ double $>($ width $)$

\}

viewportHeight $=$ static_cast $<$ double $>($ height $)$;

QQuaternion TrackBall:: getRotation()

if ( $t$ rackingMouse)

return rotation:

QTime currentTime = QTime: : currentTime ();

double angle = velocity * lastTime.msecsTo(currentTime);

return QQuaternion: : fromAxisAndAngle (axis, angle) * rotation;

QVector3D TrackBall:: mousePosTo3D (const QPointF \&p)

return QVector3D(2.0* p.x() / viewportWidth - 1.0 $1.0-2.0 * \mathrm{p} . \mathrm{y}() /$ viewportHeight,

$$
0.0) \text {; }
$$

Listing 40. File trackball.cpp.

APPENDIX E

AuXiliary Classes for PhONG Lighting Model

\# ifndef CAMERA

\#define CAMERA_H

\#include <QVector $3 D>$

class Camera

public: 


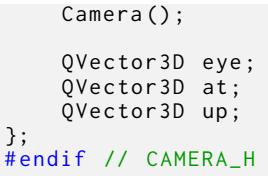

Listing 41. File camera.h

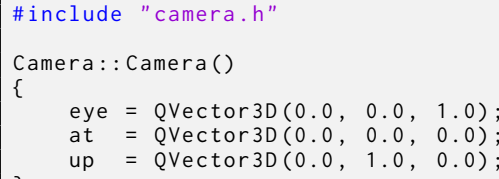

Listing 42. File camera.cpp

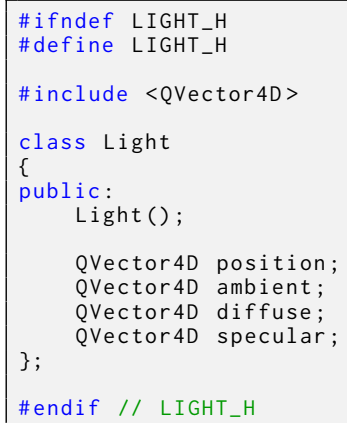

Listing 43. File light.h.

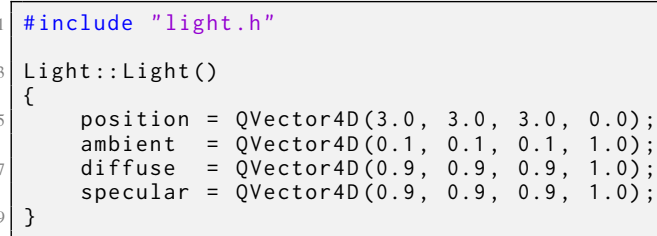

Listing 44. File light.cpp.

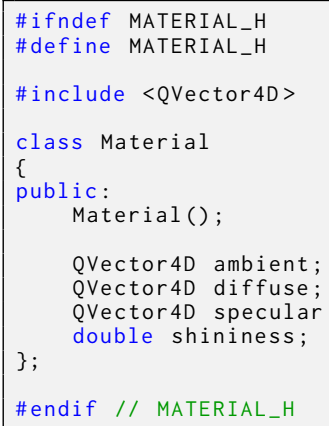

Listing 45. File material.h.

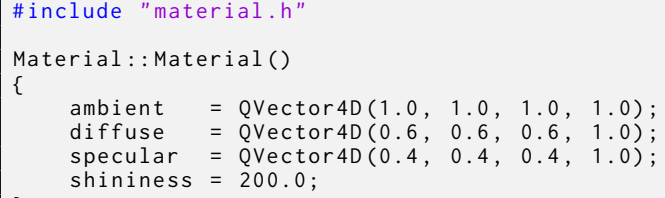

Listing 46. File material.cpp.

\section{APPENDIX F}

\section{TAKING A SCREENSHOT OF THE OPENGL SCENE}

Listing 47 presents the slot method that takes a screenshot of the OpenGL Scene. The method QGLWidget: :grabFrameBuffer() returns the contents of the frame buffer as a QImage object, which is saved to disk using the method QImage: :save().

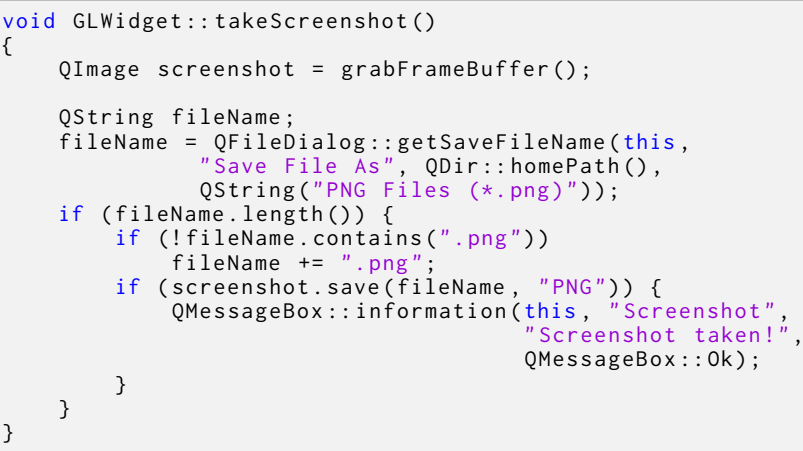

Listing 47. Slot responsible to take a screenshot of the OpenGL scene.

\section{REFERENCES}

[1] "OpenGL - The Industry Standard for High Performance Graphics," 2012. [Online]. Available: http://www.opengl.org/

[2] "GLUT - The OpenGL Utility Toolkit," 2012. [Online]. Available: http://www.opengl.org/resources/libraries/glut/

[3] Qt, "Qt-Cross-Platform application and UI framework," 2012. [Online]. Available: http://qt.nokia.com/

[4] J. Blanchette and M. Summerfield, C++ GUI Programming with Qt 4, 2nd ed. Prentice Hall, 2008.

[5] M. Summerfield, Advanced Qt Programming: Creating Great Software with $C++$ and Qt 4. Prentice Hall, 2010.

[6] Qt, "Qt in use," 2012. [Online]. Available: http://qt.nokia.com/qt-in-use

[7] - "QtOpenGL Module," 2012. [Online]. Available: http://qt-project. org/doc/qt-4.8/qtopengl.html

[8] E. Angel and D. Shreiner, Interactive Computer Graphics: A Top-Down Approach with Shader-Based OpenGL, 6th ed. Addison Wesley, 2011.

[9] M. P. B. Donald D Hearn and W. Carithers, Computer Graphics with OpenGL, 4th ed. Prentice Hall, 2010.

[10] D. Wolff, OpenGL 4.0 Shading Language Cookbook. Pactk Publishing, 2011.

[11] R. Marroquim and A. Maximo, "Introduction to GPU Programming with GLSL," in Computer Graphics and Image Processing (SIBGRAPI TUTORIALS), 2009 Tutorials of the XXII Brazilian Symposium on, oct. 2009, pp. $3-16$.

[12] Qt, "Developing Qt," 2012. [Online]. Available: http://qt-project.org/ wiki/Category:Developing_Qt

[13] -, "Qmake Variable Reference," 2012. [Online]. Available: http://qt-project.org/doc/qt-4.8/qmake-variable-reference.html

[14] - "QGLWidget Class Reference," 2012. [Online]. Available: http://qt-project.org/doc/qt-4.8/QGLWidget.html

[15] -, "Layout Management," 2012. [Online]. Available: http:// qt-project.org/doc/qt-4.8/layout.html

[16] - "Signals \& Slots," 2012. [Online]. Available: http://qt-project. org/doc/qt-4.8/signalsandslots.html

[17] "OFF File Format," 2012. [Online]. Available: http://www.geomview. org/docs/html/OFF.html

[18] E. Lengyel, Mathematics for 3D Game Programming and Computer Graphics, 3rd ed. Course Technology PTR, 2011.

[19] — , "Computing Tangent Space Basis Vectors for an Arbitrary Mesh. Terathon Software 3D Graphics Library," 2001. [Online]. Available: http://www.terathon.com/code/tangent.html

[20] Qt, “The Qt Resource System," 2012. [Online]. Available: http: //qt-project.org/doc/qt-4.8/resources.html

[21] "QFile Class Reference," 2012. [Online]. Available: http: //doc-snapshot.qt-project.org/4.8/qfile.html 
[22] M. J. Kilgard, "Modern OpenGL usage: using vertex buffer objects well," in ACM SIGGRAPH ASIA 2008 courses, ser. SIGGRAPH Asia '08. New York, NY, USA: ACM, 2008, pp. 49:1-49:19. [Online]. Available: http://doi.acm.org/10.1145/1508044.1508093

[23] Qt, "QGLBuffer Class Reference," 2012. [Online]. Available: http: //qt-project.org/doc/qt-4.8/qglbuffer.html

[24] _ "The Event System," 2012. [Online]. Available: http://qt-project. org/doc/qt-4.8/eventsandfilters.html

[25] - "QMatrix4x4 Class Reference," 2012. [Online]. Available: http://qt-project.org/doc/qt-4.8/qmatrix4x4.html

[26] _- "QGLFormat Class Reference," 2012. [Online]. Available: http://qt-project.org/doc/qt-4.8/QGLFormat.html

[27] - "Boxes I Documentation | Qt Developer Network," 2012. [Online]. Available: http://qt-project.org/doc/qt-4.8/demos-boxes.html

[28] — - "Qt3D Module," 2012. [Online]. Available: http://doc-snapshot. qt-project.org/5.0/qt3d-reference.html 